\title{
Adherence and drop-out in randomized controlled trials of exercise interventions in people with multiple sclerosis: A systematic review and meta-analyses
}

Dennett, Rachel

http://hdl.handle.net/10026.1/15703

10.1016/j.msard.2020.102169

Multiple Sclerosis and Related Disorders

Elsevier BV

All content in PEARL is protected by copyright law. Author manuscripts are made available in accordance with publisher policies. Please cite only the published version using the details provided on the item record or document. In the absence of an open licence (e.g. Creative Commons), permissions for further reuse of content should be sought from the publisher or author. 
Adherence and drop-out in randomized controlled trials of exercise interventions in people with multiple sclerosis: A systematic review and metaanalyses

Rachel Dennett (BSc)

Laurits T Madsen (MSc) $)^{b}$

Luke Connolly $(\mathrm{PhD})^{\mathrm{a}}$

Joanne Hosking $(\mathrm{PhD})^{\mathrm{c}}$

Ulrik Dalgas $(\mathrm{PhD})^{\mathrm{b}}$

Jennifer Freeman $(\mathrm{PhD})^{\mathrm{a}}$

a. School of Health Professions, Faculty of Health: Medicine, Dentistry and Human Sciences, University of Plymouth, Plymouth, UK

b. Exercise Biology, Dep. Public Health, Aarhus University, Denmark

c. Medical Statistics, Faculty of Health: Medicine, Dentistry and Human Sciences, University of Plymouth

\section{Corresponding author:}

Rachel Dennett, SF20, Peninsula Allied Health Centre, University of Plymouth, Derriford Road, Plymouth, PL6 8BH.

Email: rachel.dennett@plymouth.ac.uk Telephone: +44 1752588835 
Word Count 4850 (excluding tables and figures)

Number of tables: 2

Number of figures: 3 


\section{Abstract}

\section{Background}

The short-term benefits of exercise in people with multiple sclerosis (MS) are well established. To sustain benefits exercise needs to continue long-term. Despite important clinical implications, no systematic reviews have synthesized evidence on adherence and drop-out in MS exercise interventions.

\section{Objectives}

1) To summarize reported adherence and drop-out data from randomized controlled trials (RCTs) of exercise interventions, and 2) identify moderators related to adherence and drop-out.

\section{Methods}

Nine databases were electronically searched in October 2018. Included studies were RCTs of exercise interventions in adults with MS published from January 1993 to October 2018. Abstracts and full texts were independently screened and selected for inclusion by two reviewers. Methodological quality was assessed using the TESTEX rating scale.

\section{Results}

Ninety three articles reporting 81 studies were included. Forty one studies (51\%) reported both adherence and drop-out data during the intervention period with three (4\%) also reporting follow-up data. Of the 41 studies, $<25 \%$ pre-defined adherence or described how adherence was measured.

Meta-analyses of 59 interventions (41 studies) showed a pooled adherence estimate of $0.87(95 \% \mathrm{Cl} 0.83$ to 0.90$)$ and $0.73(\mathrm{Cl} 0.68-0.78)$ when including drop-outs. 
Mean age, proportion of females and intervention duration were inversely associated with adherence.

\section{Conclusion}

Little consensus existed on definition of adherence or determination of drop-out in MS exercise studies, with reporting generally of poor quality, if done at all. Hence it is largely unknown what can moderate adherence and whether exercise continued following an exercise intervention. Researchers should ensure clear transparent measurement and reporting of adherence and drop-out data in future trials.

Key Words: multiple sclerosis; exercise; adherence; drop-out; review PROSPERO trial register number CRD42018112866. 


\section{Introduction}

Promoting exercise in people with multiple sclerosis (pwMS) is important since the short-term benefits of exercise are well documented ${ }^{1-4}$ as are the low levels of physical activity within the population. ${ }^{5}$ The international community of researchers $^{6}$ and clinicians continue to develop our understanding of exercise in pwMS and work towards identifying the most effective exercise interventions for people with a range of disability $7,8,1,9,10$ and in a range of settings. ${ }^{11-13}$ For example, over the past twenty years interventions to enhance long-term engagement with activity ${ }^{14-16}$ have been developed and evaluated and the value of incorporating behavioural interventions to support behaviour change has been investigated. ${ }^{17-19}$ These developments are vital since sustained engagement in exercise is required to retain any benefits gained. ${ }^{20}$

A key term used when considering long-term engagement with an intervention is "adherence". Within the field of exercise research this term is often used synonymously with that of compliance, concordance or participation but within this paper the term adherence as defined by the World Health Organisation (WHO) (see definitions section below) will be used. ${ }^{21}$ It is of note that according to WHO, across diseases, adherence is the single most important modifiable factor that affects outcome. Despite the advances in MS exercise and rehabilitation research, poor exercise and physical activity levels are still reported in $\mathrm{pwMS}^{5}$, highlighting the importance of continuing to gain greater depth of understanding regarding the factors that impact adherence in this population.

In addition, in order for any intervention to have a positive long-term impact it is imperative that its efficacy transcends the research setting into clinical practice 
and the daily lives of those people it is designed to help. As such, it is important that research is conducted and reported in a manner that allows clinicians and healthcare providers to be confident in the quality, replicability and relevance of the findings. It is also important that effective exercise interventions are suitable for implementation within the financial constraints of health service provision and are feasible and acceptable to the user in order to maximise adherence.

Many of these important factors, including intervention delivery, level of supervision, study retention and intervention adherence were considered by Allen et. al. ${ }^{22}$ in a review of exercise interventions in people with Parkinson's disease. Interventions from the 53 included studies were typically of short duration and highly supervised, with less than half reporting adherence. They highlighted the challenge this presented to clinicians considering the cost-benefit balance when seeking to translate research into practice. It is not yet known whether similar issues are experienced in the field of MS or whether sufficient information exists to identify important moderators of long-term adherence and drop-out in exercise studies. Therefore, the objectives of this systematic review were to 1) summarize reported adherence and drop-out data from Randomized Controlled Trials (RCTs) of exercise interventions during the intervention and at follow up, and 2) identify moderators related to adherence and drop-out during the exercise intervention and at follow up.

\section{Methods}

This review is reported in line with the Preferred Reporting Items for Systematic Reviews and Meta- Analyses (PRISMA) ${ }^{23}$ The protocol for this review was registered with PROSPERO ref CRD42018112866. 
The following PICO question was formulated and guided the literature search and study inclusion: What adherence and drop-out data are reported by studies evaluating structured exercise interventions (according to the definition of Caspersen ${ }^{24}$ ) in pwMS, during both the intervention period and any follow-up period, and what moderators of adherence and drop-out can be identified, which relate to these exercise interventions?

\subsection{Definitions}

The following definitions are used within this review:

Exercise: As defined by Caspersen, a form of physical activity that is planned, structured and repetitive, and is undertaken with the objective of improving or maintaining at least one aspect of physical fitness; that is strength, flexibility or aerobic endurance. ${ }^{24}$

Physical activity: Any bodily movement produced by skeletal muscles that requires energy expenditure. ${ }^{24}$

Adherence: The World Health Organisation (WHO) define adherence as "the extent to which a person's behaviour; taking medication, following a diet, and/or executing lifestyle changes, corresponds with agreed recommendations from a health care provider". ${ }^{25}$ Adherence is reported as the number of attended sessions expressed as a $\%$ of the total number of planned supervised sessions, and/or the percentage of completed prescribed home programme exercises/sessions where this was a component of the intervention. In addition, adherence can be reported according to 
pre-defined cut-offs as stipulated by study authors. Where possible distinction is made between adherence to session attendance (session adherence) and adherence to the specific exercise protocol (content adherence).

Drop-out: Participants that leave a study during the intervention period or during the follow up period expressed as:

$\%$ drop-outs during intervention period $=($ drop-outs during intervention period $/$ total number recruited participants) $\times 100$

$\%$ drop-outs during follow up $=$ (drop-outs during follow up period $/$ total number recruited participants) $\times 100$

Study drop-outs could be related to a multitude of factors. Some, directly related to the exercise intervention itself such as time commitment, but others may be study related factors such as failure to attend follow up assessments

Adverse event: Any unfavourable and unintended symptom or disease that develops or worsens during the period of the trial, whether or not it is considered to be related to the trial intervention. ${ }^{26}$

\subsection{Eligibility criteria}

To be eligible for inclusion, studies were RCTs in adults over 18 years of age with a diagnosis of MS but regardless of gender, disease duration, MS phenotype or level of disability. Trials involved exercise interventions of any modality (location, group/ individual structure, level of supervision, intervention duration, session duration, intensity, frequency); content (aerobic, resistance, combined, other); with or 
without inclusion of a behavioural or home exercise component and with or without a follow up period.

Studies where the primary intention was to improve balance but the intervention was exercise (as defined by Caspersen ${ }^{24}$ ) were included. Studies reporting: balance gaming interventions (such as the Wii Fit); interventions specifically for the upper limb; gait re-education, where the primary intention was to impact on spatial or temporal parameters of gait; vestibular rehabilitation; and wheelchair propulsion interventions were not included. Studies reporting activities where the participant could be passive such as hippotherapy and robotic training were also not included. Control interventions could include passive controls, (often reported as usual activity/ care or a non-targeted exercise intervention such as relaxation or massage) or active controls where an active exercise comparator was included.

Included studies had to report at least one objective and/or self-report measure of either strength, aerobic capacity, endurance, fatigue, walking capacity or physical activity. Measures of walking capacity could include 10 metre, 25 foot, 2 minute and 6 minute walking tests and any type of accelerometry data.

Exercise interventions were categorised as either aerobic, resistance or combined training or as 'other' interventions, such as yoga, Pilates and inspiratory muscle training.

\subsection{Data sources and search strategy}


A search strategy was developed in liaison with an information specialist, based on the following key terms; "multiple sclerosis" OR MS AND exercise OR "physical activity" AND strength OR aerobic OR fitness OR training. The full search strategy can be found in appendix 1. Two reviewers (LM and RD) conducted electronic searches of MEDLINE, EMBASE, CINAHL, AMED, PEDro, SPORTDiscus, PsycINFO, Web of Sciences and SCOPUS from January 1993 to October 2018. This 25 year period was chosen to encompass the earliest of MS rehabilitation/ exercise randomized controlled studies. Electronic searches were supplemented by hand searches of reference lists. Duplicates were removed and records were imported into the Rayyan data management system. Titles and abstracts were independently screened for eligibility based on the inclusion criteria by two reviewers (RD or JF and LM). Finally, full texts of remaining articles were read by two reviewers (LM, LC or RD) and any disagreements were discussed with a fourth member of the research team (JF).

\subsection{Data extraction}

A customized Excel spreadsheet was used to collate the extracted data from included studies. Details extracted included participant characteristics (age, gender, disease duration, MS phenotype, disability level and fatigue as a symptom); modality of the intervention (setting, group/ individual structure, level of supervision, intervention duration, session duration, intensity, frequency); content of the intervention (aerobic/ resistance/ combined / other modality/ including a behavioral or home exercise program component); report of adverse events, \% drop-out, and adherence during the intervention period and at any follow up. Where there was 
missing data, the median was used as a proxy for the mean and 0.75 times the interquartile range or 0.25 times the range as proxies for the standard deviation. ${ }^{27}$ Standard errors were converted into standard deviations by multiplying the standard error by the square root of the sample size. ${ }^{27}$

Data extraction was completed by one reviewer (RD, LM or LC) and $10 \%$ of papers $(n=10)$ were also extracted by a second reviewer for quality assurance purposes (JF or UD) with a kappa of $0.639, p<0.0001$ indicating substantial agreement between reviewers. Adherence data from all studies that reported this was extracted by two reviewers (RD, LM or LC).

\subsection{Quality Assessment}

Methodological quality was assessed independently by two reviewers (LM, LC or RD) using the Tool for the assEssment of Study qualiTy and reporting in EXercise (TESTEX) rating scale; a novel tool designed for appraising methodological quality of exercise studies. ${ }^{28}$ Any discrepancies were discussed, and on six occasions a third reviewer (JF) was consulted to reach consensus.

\subsection{Synthesis of results}

The adherence data were extracted in the form of proportion of participants 'adherent' to an intervention or mean number or percentage of sessions attended. Where adherence was reported as mean number or percentage of sessions attended, this was not always accompanied by a measure of variation.

Consequently, and in line with McPhate et al. ${ }^{29}$ the data were converted to represent 
the proportion of participants 'adherent' in order to include all interventions in the analysis. In studies with an exercise comparator group, adherence data were reported separately for each different intervention evaluated in the same study; for example, Pilutti et al. 2016a (recumbent stepper), Pilutti et al. 2016b bodyweight support treadmill). For the purpose of clear reporting, in cases where study results were reported by more than one article, the paper reporting the primary outcome or with most complete adherence data was used.

A meta-analysis was conducted to estimate the pooled adherence across the 59 interventions (41 studies) using the Freeman-Tukey double arcsine transformation to transform the raw proportions. We hypothesised that intervention type (i.e. 'aerobic', 'resistance', 'combined' or 'other) would be a moderator of adherence and as such we calculated pooled adherence within these sub-groups as well as across all studies, taking into account this sub-grouping using a mixed effects model.

Heterogeneity was assessed using the I-squared statistic and metaregression was used to examine the association between the a priori defined study intervention related variables (potential moderators including: intervention type, duration, and frequency, supervised or unsupervised, inclusion of a behavioural intervention or home exercise component and EDSS, disease duration, mean age and proportion of female participants, TESTEX score of study quality) and adherence.

Studies reported adherence data for participants who completed an intervention. As such, this did not take into account participants who dropped out of a study (for whatever reason). In this review therefore, in order to consider the impact 
of study drop-outs on adherence, analysis was repeated with percentage adherence recalculated to include drop-outs. Mindful that reasons for drop-out (exercise intervention or study process related) were not consistently provided, each drop-out was assumed a conservative adherence estimate of $0 \%$.

A further meta-analysis was also carried out including only those studies which reported mean adherence (with a measure of variation). All analyses were carried out using the 'metafor' 30 package in $\mathrm{R} .{ }^{31}$

\section{Results}

\subsection{Study characteristics}

Searches identified 10267 articles which after deduplication resulted in 6612 titles and abstracts being reviewed. Of these, 133 were included for full text review of which 93 met the inclusion criteria. For further detail please refer to figure 1.

Insert Figure 1

PRISMA flow diagram here 
Figure 1: PRISMA Flow Diagram
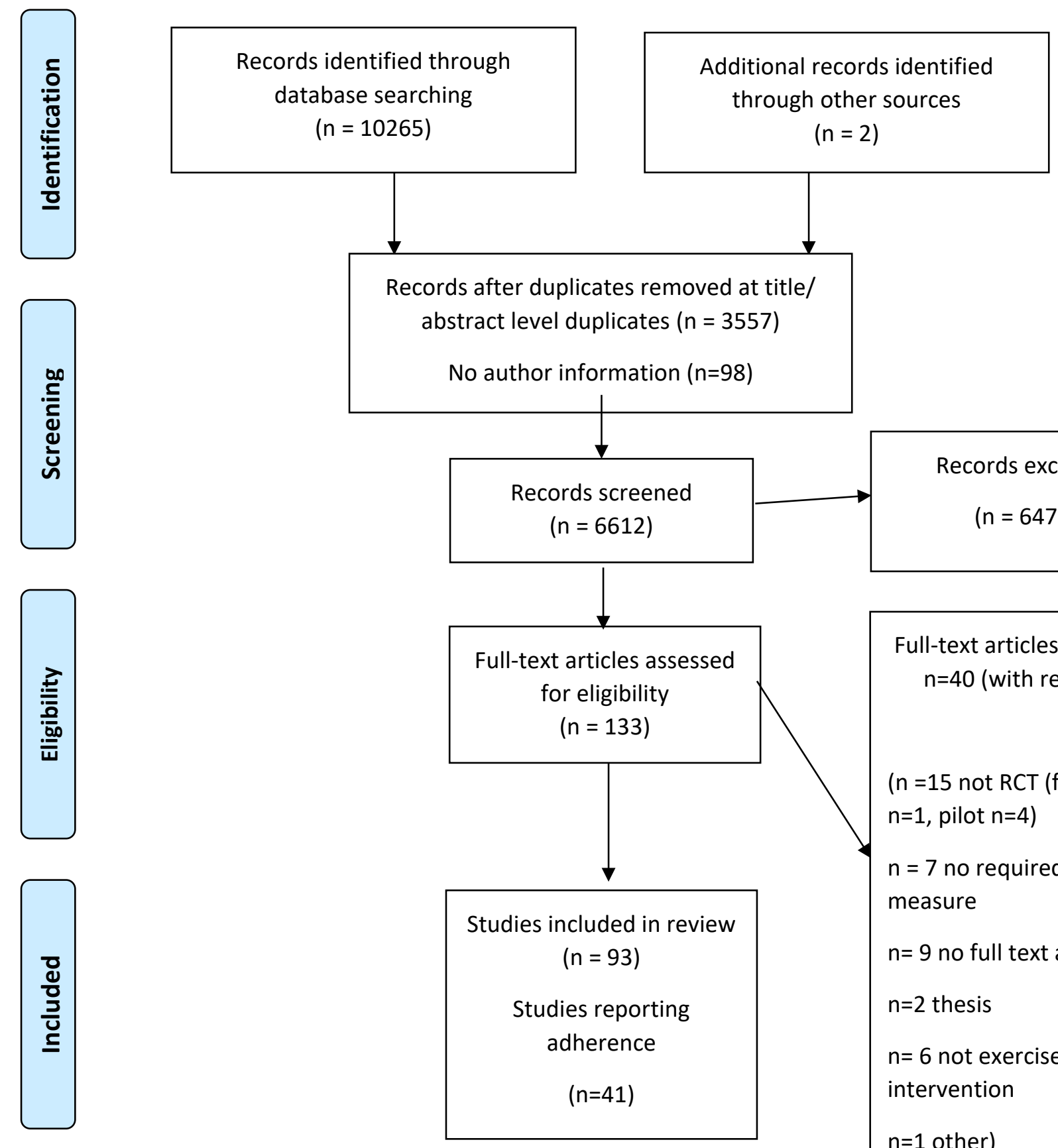

Records excluded

$(n=6479)$

Full-text articles excluded $n=40$ (with reasons)

( $\mathrm{n}=15$ not RCT (feasibility $n=1$, pilot $n=4$ )

$\mathrm{n}=7$ no required outcome measure $\mathrm{n}=9$ no full text available $\mathrm{n}=2$ thesis $\mathrm{n}=6$ not exercise intervention $\mathrm{n}=1$ other) 
The 93 included articles reported on 81 RCTs which involved 4007 pwMS, mean (SD) age 43.8 (8.2) years, disease duration 9.2 (6.3) years. Eleven of the included studies only involved people with relapsing remitting MS (RRMS), 19 included people with all types of MS (RRMS, secondary progressive MS, primary progressive MS and benign) and 11 did not state MS type. Disability level was reported using the Expanded Disability Status Scale (EDSS) in 59 studies. ${ }^{32}$ Other measures used were the Patient Determined Disease Steps (PDDS) ${ }^{33}$ and the Guys Neurological Disability Scale (GNDS) ${ }^{34}$ The vast majority of studies included people who were ambulant with or without walking aids (EDSS 0-6.5) with only three studies $^{35-37}$ including participants with an EDSS $\geq 7$.

In eleven studies the RCT involved exercise comparator groups, seven included active controls and 31 enrolled passive controls. Of the passive controls, two were defined as relaxation exercises ${ }^{41,90}$ and one as massage. ${ }^{92}$ The remaining studies described the content of the passive control as "usual activity" or "usual care". Two studies gave some information about what usual activity could comprise of, for example 'recording in a diary all physical activity exceeding 20 minutes and occurring more than twice a week', ${ }^{58}$ or 'usual care could include habitual exercise participants engaged in, or therapy, provided it did not include progressive resistance training. ${ }^{59}$ Importantly however, no authors defined, detailed or reported what usual activity included or stated adherence to that activity. As such it is not possible to comment on adherence to the passive control group content. Adherence data throughout this review, therefore, is reported for active exercise interventions, both the exercise comparator and active control groups. 


\subsection{Studies reporting adherence and drop-out}

Of the 81 included studies, $41(51 \%)$ reported adherence to an intervention (exercise comparator and/ or active control). Details regarding the study interventions can be found in table 1. The definitions of adherence used and methods of reporting were not consistent. In 38 cases adherence was defined as the proportion of prescribed sessions attended. On three occasions adherence was defined as the proportion of people who were adherent based upon a pre-defined cut-off; exercise on $45 \%^{38}$ of days during the study or $67 \%{ }^{39}$ or $80 \%{ }^{40}$ of sessions attended.

Nine ${ }^{11,13,40-46}$ papers stated within their methods section that adherence was an outcome of interest. Sample size in the studies reporting adherence ranged from $n=14^{47}$ to $n=314^{9}$ with mean (SD) intervention participant age $46.1(8.4)$ years and control participant age 45.5 (7.8) years. Mean disease (SD) duration of intervention participants was 9.4 (7.1) years and EDSS score 3.8 (1.2) and controls was 9.5 (6.2) years and 3.3 (1.1) EDSS score.

Mean intervention duration was 12.2 weeks (range 3-26) and mean frequency 3.3 sessions a week (range 1-7). Of the included studies only $11(14 \%)$ included a follow up assessment (range 4-26 weeks) of which three (4\%) made reference to exercise adherence during this period.

Insert table 1 here 
Table 1: Characteristics of included studies reporting adherence

\begin{tabular}{|c|c|c|c|c|c|c|c|c|c|c|c|c|c|c|c|}
\hline $\begin{array}{l}\frac{n}{2} \\
\frac{1}{2}\end{array}$ & 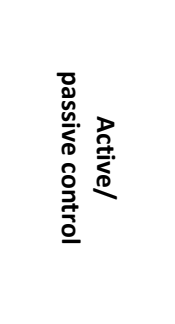 & 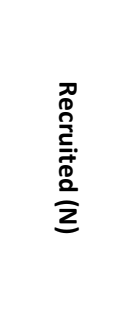 & 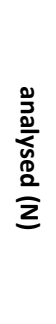 & 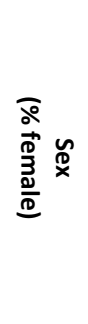 & 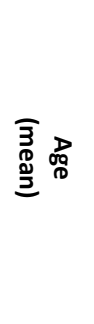 & 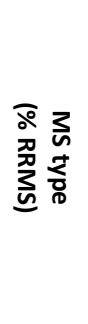 & 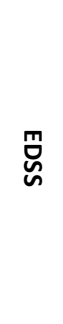 & 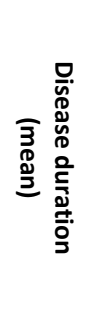 & 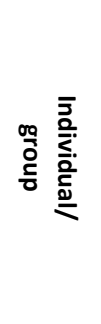 & 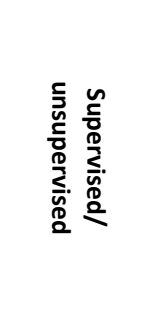 & 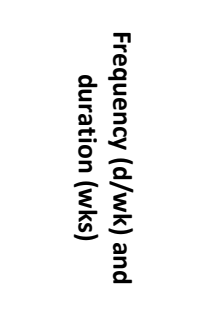 & 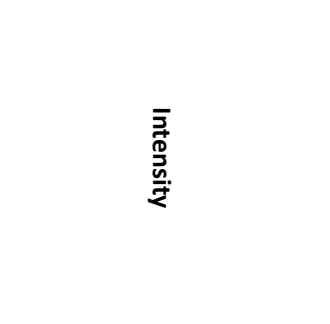 & 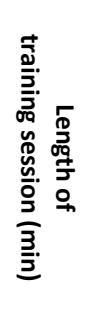 & 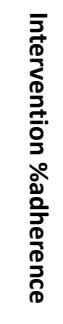 & 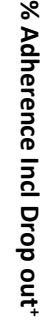 \\
\hline \multicolumn{16}{|l|}{ Aerobic } \\
\hline \multicolumn{16}{|l|}{$\begin{array}{c}\text { Bicycle } \\
\text { ergometer }\end{array}$} \\
\hline $\begin{array}{l}\text { Collett, } \\
2010^{48 *}\end{array}$ & $\begin{array}{l}\text { Three } \\
\text { comparator } \\
\text { groups }\end{array}$ & $\begin{array}{l}\text { Int 1: } 20 \\
\text { Int 2: } 21 \\
\text { Int 3: } 20\end{array}$ & $\begin{array}{l}20 \\
18 \\
17\end{array}$ & $\begin{array}{l}53 \\
80 \\
78\end{array}$ & $\begin{array}{l}55.0 \\
52.0 \\
50.0\end{array}$ & 40 & NR & $\begin{array}{l}12.0 \\
15.0 \\
11.0\end{array}$ & $\begin{array}{l}N R \\
N R \\
N R\end{array}$ & $\begin{array}{l}\text { Supervised } \\
\text { Supervised } \\
\text { Supervised }\end{array}$ & $\begin{array}{l}2 d / w k^{*} 12 w k s \\
2 d / w k^{*} 12 w k s \\
2 d / w k^{*} 12 w k s\end{array}$ & $\begin{array}{l}\text { Continuous: } 45 \% \text { PPO } \\
\text { Interval: } 30 \text { s on } / 30 \text { s off } \\
\text { at } 90 \% \text { of PPO } \\
\text { Combined: } 10 \text { min HIIT } \\
+10 \text { min continuous }\end{array}$ & $\begin{array}{l}20 \\
20 \\
20\end{array}$ & $\begin{array}{l}66 \\
54 \\
50\end{array}$ & $\begin{array}{l}56 \\
51 \\
45\end{array}$ \\
\hline $\begin{array}{l}\text { Mostert, } \\
2002^{81} \\
\text { (upper- and } \\
\text { lower-limb } \\
\text { cycling) }\end{array}$ & $\begin{array}{l}\text { Active } \\
\text { control } \\
\text { (inpatient } \\
\text { physical } \\
\text { therapy) }\end{array}$ & $\begin{array}{l}\text { Int: } 18 \\
\text { Con: } 18\end{array}$ & $\begin{array}{l}13 \\
13\end{array}$ & $\begin{array}{l}77 \\
85\end{array}$ & $\begin{array}{l}45.2 \\
43.9\end{array}$ & $\begin{array}{l}31 \\
39\end{array}$ & $\begin{array}{l}4.6 \\
4.5\end{array}$ & $\begin{array}{l}11.2 \\
12.6\end{array}$ & $\begin{array}{l}\text { NR } \\
N R\end{array}$ & $\begin{array}{c}\text { Supervised } \\
\text { NR }\end{array}$ & $\begin{array}{c}5 \mathrm{~d} / \mathrm{wk}^{*} 4 \mathrm{wk} \\
\mathrm{NR}\end{array}$ & $\begin{array}{l}\text { NR } \\
\text { NR }\end{array}$ & $\begin{array}{l}30 \\
N R\end{array}$ & $\begin{array}{l}65 \\
\text { NR }\end{array}$ & $\begin{array}{l}47 \\
\text { NR }\end{array}$ \\
\hline \multirow[t]{2}{*}{$\begin{array}{l}\text { Negaresh, } \\
2018^{82}\end{array}$} & $\begin{array}{c}\text { Passive } \\
\text { control } \\
\text { (not defined } \\
\text { usual } \\
\text { activity) }\end{array}$ & $\begin{array}{l}\text { Int: } 36 \\
\text { Con: } 30\end{array}$ & $\begin{array}{l}34 \\
27\end{array}$ & $\begin{array}{l}64 \\
67\end{array}$ & $\begin{array}{l}31.7 \\
30.6\end{array}$ & 100 & $\begin{array}{l}1.7 \\
1.5\end{array}$ & $\begin{array}{l}7.3 \\
7.4\end{array}$ & $\begin{array}{l}N R \\
N R\end{array}$ & $\begin{array}{c}\text { Supervised } \\
\text { NR }\end{array}$ & $\begin{array}{c}3 \mathrm{~d} / \mathrm{wk}^{*} 8 \mathrm{wk} \\
\mathrm{NR}\end{array}$ & $\begin{array}{c}\text { Intermittent: } 2 \mathrm{~min} \\
\text { on } / 2 \text { min off at } 60-75 \% \\
\text { of PPO } \\
\text { NR }\end{array}$ & $\begin{array}{c}42-66 \\
N R\end{array}$ & $\begin{array}{l}92 \\
\text { NR }\end{array}$ & $\begin{array}{l}87 \\
\text { NR }\end{array}$ \\
\hline & $\begin{array}{c}\text { Comparator } \\
\text { (yoga) }\end{array}$ & Int1: 21 & 15 & 87 & 48.8 & NR & 2.9 & NR & Group & Supervised & $1 d / w k^{*} 26 w k s$ & NR & NR & 65 & 46 \\
\hline \multirow[t]{2}{*}{ Oken, $2004^{44}$} & Passive & Int2: 26 & 22 & 91 & 49.8 & NR & 3.2 & NR & Group & Supervised & $1 \mathrm{~d} / \mathrm{wk}^{*} 26 \mathrm{wks}$ & NR & 90 & 68 & 58 \\
\hline & $\begin{array}{c}\text { Control (not } \\
\text { defined }\end{array}$ & Con: 22 & 20 & 100 & 48.4 & $N R$ & 3.1 & $N R$ & NR & NR & NR & NR & $N R$ & NR & NR \\
\hline
\end{tabular}




\begin{tabular}{|c|c|c|c|c|c|c|c|c|c|c|c|c|c|c|c|}
\hline \multirow[b]{2}{*}{$\begin{array}{l}\text { Petajan, } \\
1996^{83} \\
\text { (combined } \\
\text { arm and leg } \\
\text { cycle } \\
\text { ergometer) }\end{array}$} & $\begin{array}{l}\text { waitlist } \\
\text { control) }\end{array}$ & & & & & & & & & & & & & & \\
\hline & $\begin{array}{c}\text { Passive } \\
\text { control } \\
\text { (not defined } \\
\text { usual } \\
\text { activity) }\end{array}$ & $\begin{array}{l}\text { Int: } 27 \\
\text { Con: } 27\end{array}$ & $\begin{array}{l}21 \\
25\end{array}$ & $\begin{array}{l}71 \\
67\end{array}$ & $\begin{array}{l}41.1 \\
39.0\end{array}$ & NR & $\begin{array}{l}3.8 \\
2.9\end{array}$ & $\begin{array}{l}9.3 \\
6.2\end{array}$ & $\begin{array}{l}\text { NR } \\
\text { NR }\end{array}$ & $\begin{array}{c}\text { Supervised } \\
\text { NR }\end{array}$ & $\begin{array}{c}3 \mathrm{~d} / \mathrm{wk} * 15 \mathrm{wk} \\
\mathrm{NR}\end{array}$ & $\begin{array}{c}60 \% \text { of } \mathrm{VO}_{2 \max } \\
\mathrm{NR}\end{array}$ & $\begin{array}{c}45-50 \\
\text { NR }\end{array}$ & $\begin{array}{l}97 \\
\text { NR }\end{array}$ & $\begin{array}{l}75 \\
\text { NR }\end{array}$ \\
\hline $\begin{array}{l}\text { Zimmer, } \\
2017^{13} \\
\text { (HIT) }\end{array}$ & $\begin{array}{c}\text { Comparator } \\
\text { (moderate } \\
\text { intensity } \\
\text { cycling) }\end{array}$ & $\begin{array}{l}\text { Int 1: } 29 \\
\text { Int 2: } 31\end{array}$ & $\begin{array}{l}27 \\
31\end{array}$ & $\begin{array}{l}74 \\
60\end{array}$ & $\begin{array}{l}51.0 \\
48.0\end{array}$ & $\begin{array}{l}52 \\
53\end{array}$ & $\begin{array}{l}4.4 \\
4.4\end{array}$ & $\begin{array}{l}12.0 \\
12.3\end{array}$ & $\begin{array}{l}\text { NR } \\
\text { NR }\end{array}$ & $\begin{array}{l}\text { Supervised } \\
\text { Supervised }\end{array}$ & $\begin{array}{l}5 d / w k * 3 w k \\
5 d / w k * 3 w k\end{array}$ & $\begin{array}{c}\text { Interval: } 5 \times 3 \mathrm{~min} \\
\text { on/ } 1.5 \mathrm{~min} \text { off at } 80 \% \\
\text { of } \mathrm{VO}_{2 \text { peak }} \\
\text { Continuous: at } 65 \% \text { of } \\
\mathrm{VO}_{2 \text { peak }}\end{array}$ & $\begin{array}{l}20 \\
30\end{array}$ & $\begin{array}{l}100 \\
100\end{array}$ & $\begin{array}{l}93 \\
97\end{array}$ \\
\hline Running & & & & & & & & & & & & & & & \\
\hline Feys, $2017^{12}$ & $\begin{array}{l}\text { Passive } \\
\text { control } \\
\text { (not defined } \\
\text { waitlist } \\
\text { control) }\end{array}$ & $\begin{array}{l}\text { Int: } 21 \\
\text { Con: } 21\end{array}$ & $\begin{array}{l}18 \\
17\end{array}$ & $\begin{array}{l}95 \\
86\end{array}$ & $\begin{array}{l}36.6 \\
44.4\end{array}$ & NR & NR & $\begin{array}{l}8.1 \\
9.2\end{array}$ & $\begin{array}{l}\text { Indiv } \\
\text { NR }\end{array}$ & $\begin{array}{c}\text { Unsupervised } \\
\text { NR }\end{array}$ & $\begin{array}{c}3 d / w k * 12 w k \\
N R\end{array}$ & $\begin{array}{l}\text { NR } \\
\text { NR }\end{array}$ & $\begin{array}{l}\text { NR } \\
\text { NR }\end{array}$ & $\begin{array}{l}94 \\
\text { NR }\end{array}$ & $\begin{array}{l}81 \\
N R\end{array}$ \\
\hline $\begin{array}{l}\text { Recumbent } \\
\text { stepper }\end{array}$ & & & & & & & & & & & & & & & \\
\hline Pilutti, $2016^{37}$ & $\begin{array}{l}\text { Comparator } \\
\text { (body weight } \\
\text { support } \\
\text { treadmill) }\end{array}$ & $\begin{array}{l}\text { Int 1: } 6 \\
\text { Int 2: } 6\end{array}$ & $\begin{array}{l}5 \\
5\end{array}$ & $\begin{array}{l}60 \\
40\end{array}$ & $\begin{array}{l}58.8 \\
48.2\end{array}$ & $\begin{array}{l}0 \\
0\end{array}$ & $\begin{array}{l}7.0 \\
7.0\end{array}$ & $\begin{array}{l}15.2 \\
12.7\end{array}$ & $\begin{array}{l}\text { NR } \\
\text { NR }\end{array}$ & $\begin{array}{l}\text { Supervised } \\
\text { Supervised }\end{array}$ & $\begin{array}{l}3 d / w k * 12 w k \\
3 d / w k * 12 w k\end{array}$ & $\begin{array}{l}\text { NR } \\
\text { NR }\end{array}$ & $\begin{array}{c}\text { Gradually } \\
\text { increased to } \\
30\end{array}$ & $\begin{array}{l}89 \\
89\end{array}$ & $\begin{array}{l}74 \\
74\end{array}$ \\
\hline Resistance & & & & & & & & & & & & & & & \\
\hline Calisthenics & & & & & & & & & & & & & & & \\
\hline $\begin{array}{l}\text { Forsberg, } \\
2016^{84}\end{array}$ & $\begin{array}{l}\text { Passive } \\
\text { control } \\
\text { (not defined } \\
\text { waitlist } \\
\text { control) }\end{array}$ & $\begin{array}{l}\text { Int: } 44 \\
\text { Con: } 43\end{array}$ & $\begin{array}{l}35 \\
38\end{array}$ & $\begin{array}{l}80 \\
82\end{array}$ & $\begin{array}{l}52.0 \\
56.3\end{array}$ & $\begin{array}{l}57 \\
34\end{array}$ & $\begin{array}{l}N R \\
N R\end{array}$ & $\begin{array}{l}15 \\
16\end{array}$ & $\begin{array}{c}\text { Group } \\
\text { NR }\end{array}$ & $\begin{array}{c}\text { Supervised } \\
\text { NR }\end{array}$ & $\begin{array}{c}2 d / w k^{*} 7 w k \\
N R\end{array}$ & $\begin{array}{l}N R \\
N R\end{array}$ & $\begin{array}{c}50-60 \\
N R\end{array}$ & $\begin{array}{l}86 \\
N R\end{array}$ & $\begin{array}{l}68 \\
\text { NR }\end{array}$ \\
\hline
\end{tabular}




\begin{tabular}{|c|c|c|c|c|c|c|c|c|c|c|c|c|c|c|c|}
\hline \multicolumn{16}{|l|}{$\begin{array}{l}\text { Progressive } \\
\text { resistance } \\
\text { training }\end{array}$} \\
\hline $\begin{array}{l}\text { Dalgas, } \\
2009^{50 *}\end{array}$ & $\begin{array}{l}\text { Passive } \\
\text { control } \\
\text { (waitlist } \\
\text { control, not } \\
\text { defined } \\
\text { usual } \\
\text { activity) }\end{array}$ & $\begin{array}{l}\text { Int: } 19 \\
\text { Con: } 19\end{array}$ & $\begin{array}{l}15 \\
16\end{array}$ & $\begin{array}{l}66 \\
62\end{array}$ & $\begin{array}{l}47.7 \\
50.4\end{array}$ & 100 & $\begin{array}{l}3.7 \\
3.9\end{array}$ & $\begin{array}{l}6.6 \\
8.1\end{array}$ & $\begin{array}{c}\text { Group } \\
\text { NR }\end{array}$ & $\begin{array}{c}\text { Supervised } \\
\text { NR }\end{array}$ & $\begin{array}{c}2 d / w^{*} 12 w k \\
N R\end{array}$ & $\begin{array}{c}\text { 3-4 sets of 8-12 } \\
\text { repetitions at 8-15 RM } \\
\text { NR }\end{array}$ & $\begin{array}{l}N R \\
N R\end{array}$ & $\begin{array}{l}100 \\
N R\end{array}$ & $\begin{array}{r}79 \\
N R\end{array}$ \\
\hline $\begin{array}{l}\text { DeBolt, } \\
2004^{11}\end{array}$ & $\begin{array}{c}\text { Passive } \\
\text { control } \\
\text { (not defined } \\
\text { usual } \\
\text { activity) }\end{array}$ & $\begin{array}{l}\text { Int: } 19 \\
\text { Con: } 18\end{array}$ & $\begin{array}{l}19 \\
17\end{array}$ & $\begin{array}{l}79 \\
78\end{array}$ & $\begin{array}{l}51.6 \\
47.8\end{array}$ & $\begin{array}{l}47 \\
44\end{array}$ & $\begin{array}{l}4.0 \\
3.5\end{array}$ & $\begin{array}{l}15.1 \\
13.0\end{array}$ & $\begin{array}{l}\text { Indiv } \\
\text { NR }\end{array}$ & $\begin{array}{c}\text { Unsupervised } \\
\text { NR }\end{array}$ & $\begin{array}{c}3 d / w k^{*} 8 w k \\
N R\end{array}$ & $\begin{array}{l}2-3 \text { sets of } 8-12 \\
\text { repetitions wearing a } \\
\text { weighted vest ( } 0.5 \% \text { of } \\
\text { BW) increasing by } 0.5- \\
1.5 \% \text { of BW every } 2 \text { wk }\end{array}$ & $\begin{array}{c}35-50 \\
N R\end{array}$ & $\begin{array}{l}95 \\
N R\end{array}$ & $\begin{array}{l}95 \\
\text { NR }\end{array}$ \\
\hline Dodd, $2011^{59}$ & $\begin{array}{l}\text { Passive } \\
\text { control } \\
\text { (Usual } \\
\text { activity + } \\
\text { social } \\
\text { program) }\end{array}$ & $\begin{array}{l}\text { Int: } 39 \\
\text { Con: } 37\end{array}$ & $\begin{array}{l}36 \\
35\end{array}$ & $\begin{array}{l}72 \\
74\end{array}$ & $\begin{array}{l}47.7 \\
50.4\end{array}$ & 100 & NR & NR & $\begin{array}{l}\text { Group } \\
\text { Group }\end{array}$ & $\begin{array}{l}\text { Supervised } \\
\text { Supervised }\end{array}$ & $\begin{array}{l}2 d / w k^{*} 10 w k \\
1 d / w k^{*} 10 w k\end{array}$ & $\begin{array}{c}2 \text { sets of } 10-12 \\
\text { repetitions at } 10-12 \text { RM } \\
\text { NR }\end{array}$ & $\begin{array}{l}45 \\
60\end{array}$ & $\begin{array}{l}92 \\
N R\end{array}$ & $\begin{array}{l}85 \\
N R\end{array}$ \\
\hline $\begin{array}{l}\text { Fimland, } \\
2010^{47}\end{array}$ & $\begin{array}{c}\text { Active } \\
\text { Control } \\
\text { (conventional } \\
\text { rehabilitation) }\end{array}$ & $\begin{array}{l}\text { Int: } 7 \\
\text { Con: } 7\end{array}$ & $\begin{array}{l}7 \\
7\end{array}$ & $\begin{array}{l}57 \\
57\end{array}$ & $\begin{array}{l}53.0 \\
54.0\end{array}$ & NR & $\begin{array}{l}4.6 \\
3.5\end{array}$ & $\begin{array}{l}8.0 \\
8.0\end{array}$ & $\begin{array}{l}\text { Indiv } \\
\text { NR }\end{array}$ & $\begin{array}{c}\text { Supervised } \\
\text { NR }\end{array}$ & $\begin{array}{c}5 d / w k * 3 w k \\
N R\end{array}$ & $\begin{array}{c}4 \text { sets of } 4 \text { repetitions } \\
\text { at } 85-90 \% \text { of } 1 R M \\
\text { NR }\end{array}$ & NR & $\begin{array}{l}100 \\
N R\end{array}$ & $\begin{array}{l}100 \\
N R\end{array}$ \\
\hline $\begin{array}{l}\text { Hosseini, } \\
2018^{39}\end{array}$ & $\begin{array}{l}\text { Comparator } \\
\text { Yoga } \\
\text { Passive } \\
\text { control } \\
\text { (not defined } \\
\text { usual } \\
\text { activity) }\end{array}$ & $\begin{array}{l}\text { Int 1: } 9 \\
\text { Int 2: } 9 \\
\text { Con: } 8\end{array}$ & $\begin{array}{l}8 \\
8 \\
8\end{array}$ & $\begin{array}{l}55 \\
55 \\
50\end{array}$ & $\begin{array}{l}32.9 \\
31.3 \\
33.0\end{array}$ & NR & NR & $\mathrm{NR}$ & $\begin{array}{l}\text { Indiv } \\
\text { Indiv } \\
\text { NR }\end{array}$ & $\begin{array}{c}\text { Unsupervised } \\
\text { Unsupervised } \\
\text { NR }\end{array}$ & $\begin{array}{c}3 d / w k^{*} 8 w k \\
3 d / w k * 8 w k \\
N R\end{array}$ & $\begin{array}{c}1 \% \text { of BW fastened to } \\
\text { body increasing by } 0.5- \\
1 \% \text { every } 2 \mathrm{wk} \\
\mathrm{NR} \\
\mathrm{NR}\end{array}$ & $\begin{array}{c}35-50 \\
60-70 \\
N R\end{array}$ & $\begin{array}{l}100 \\
100 \\
N R\end{array}$ & $\begin{array}{l}89 \\
89 \\
\text { NR }\end{array}$ \\
\hline $\begin{array}{l}\text { Harvey, } \\
1999^{56}\end{array}$ & $\begin{array}{l}\text { Comparator } \\
\text { (mobility } \\
\text { exercises) } \\
\text { Passive } \\
\text { control (not } \\
\text { defined, }\end{array}$ & $\begin{array}{l}\text { Int 1: } 7 \\
\text { Int 2: } 7 \\
\text { Con: } 5\end{array}$ & $\begin{array}{l}6 \\
5 \\
5\end{array}$ & $\begin{array}{l}83 \\
83 \\
80\end{array}$ & $\begin{array}{l}38.0 \\
49.0 \\
43.0\end{array}$ & 100 & NR & $\begin{array}{c}5 \\
5 \\
10\end{array}$ & $\begin{array}{l}\text { Indiv } \\
\text { Indiv } \\
\text { NR }\end{array}$ & $\begin{array}{c}\text { Unsupervised } \\
\text { Unsupervised } \\
\text { NR }\end{array}$ & $\begin{array}{c}2 d / * 8 w k \\
7 d / w k * 8 w k \\
N R\end{array}$ & $\begin{array}{c}5 \text { sets of } 10 \text { leg } \\
\text { extensions using } 0.5 \text { or } \\
1 \mathrm{~kg} \text { ankle weights } \\
\text { NR } \\
\text { NR }\end{array}$ & $\begin{array}{l}\text { NR } \\
\text { NR } \\
\text { NR }\end{array}$ & $\begin{array}{l}69 \\
69 \\
N R\end{array}$ & $\begin{array}{l}59 \\
59 \\
N R\end{array}$ \\
\hline
\end{tabular}




\begin{tabular}{|c|c|c|c|c|c|c|c|c|c|c|c|c|c|c|c|}
\hline & $\begin{array}{c}\text { usual } \\
\text { activity) }\end{array}$ & & & & & & & & & & & & & & \\
\hline $\begin{array}{l}\text { Kjolhede, } \\
2015^{51 *}\end{array}$ & $\begin{array}{c}\text { Passive } \\
\text { control } \\
\text { (waitlist not } \\
\text { defined } \\
\text { usual } \\
\text { activity) }\end{array}$ & $\begin{array}{l}\text { Int: } 18 \\
\text { Con: } 17\end{array}$ & $\begin{array}{l}17 \\
15\end{array}$ & NR & 43.2 & 100 & 3 & 5 & $\begin{array}{l}\text { NR } \\
\text { NR }\end{array}$ & $\begin{array}{c}\text { Supervised } \\
\text { NR }\end{array}$ & $\begin{array}{c}2 \mathrm{~d} / \mathrm{wk}^{*} \\
24 \mathrm{wk} \\
\mathrm{NR}\end{array}$ & $\begin{array}{c}\text { 3-5 sets of 6-10 } \\
\text { repetitions at 6-15 RM } \\
\text { NR }\end{array}$ & $\begin{array}{l}\mathrm{NR} \\
\mathrm{NR}\end{array}$ & $\begin{array}{l}93 \\
N R\end{array}$ & $\begin{array}{l}88 \\
N R\end{array}$ \\
\hline $\begin{array}{l}\text { Manca, } \\
2017^{85} \\
\text { Contra lateral } \\
\text { strength } \\
\text { training }\end{array}$ & $\begin{array}{l}\text { Active } \\
\text { control } \\
\text { (direct } \\
\text { strength } \\
\text { training) }\end{array}$ & $\begin{array}{l}\text { Int 1: } 15 \\
\text { Int 2: } 15\end{array}$ & $\begin{array}{l}15 \\
15\end{array}$ & $\begin{array}{l}64 \\
75\end{array}$ & $\begin{array}{l}42.1 \\
47.3\end{array}$ & $\begin{array}{l}100 \\
100\end{array}$ & $\begin{array}{l}3.8 \\
4.1\end{array}$ & $\begin{array}{l}12.7 \\
16.8\end{array}$ & $\begin{array}{l}\text { Indiv } \\
\text { Indiv }\end{array}$ & $\begin{array}{l}\text { Supervised } \\
\text { Supervised }\end{array}$ & $\begin{array}{l}3 d / w k^{*} 6 w k \\
3 d / w k^{*} 6 w k\end{array}$ & $\begin{array}{c}3 \text { sets of } 4 \text { repetitions } \\
\text { at } 45 \% \mathrm{~s} \text { and } 10 \% \text { s at } \\
100 \% \text { of } 1 \mathrm{RM} \\
3 \text { sets of } 4 \text { repetitions } \\
\text { at } 45 \% \text { and } 10 \% \text { s at } \\
100 \% \text { of } 1 \mathrm{RM}\end{array}$ & $\begin{array}{l}25 \\
25\end{array}$ & $\begin{array}{l}91 \\
91\end{array}$ & $\begin{array}{l}91 \\
91\end{array}$ \\
\hline $\begin{array}{l}\text { Medina- } \\
\text { Perez, 2014 }\end{array}$ & $\begin{array}{c}\text { Passive } \\
\text { control } \\
\text { (not defined } \\
\text { usual } \\
\text { activity) }\end{array}$ & $\begin{array}{l}\text { Int: } 30 \\
\text { Con: } 12\end{array}$ & $\begin{array}{l}30 \\
12\end{array}$ & $\begin{array}{l}88 \\
71\end{array}$ & $\begin{array}{l}49.6 \\
46.2\end{array}$ & $\begin{array}{l}100 \\
100\end{array}$ & $\begin{array}{l}4.5 \\
4.1\end{array}$ & $\begin{array}{l}11.3 \\
12.2\end{array}$ & $\begin{array}{l}\text { NR } \\
\text { NR }\end{array}$ & $\begin{array}{c}\text { Supervised } \\
\text { NR }\end{array}$ & $\begin{array}{c}2 \mathrm{~d} / \mathrm{wk}^{*} 12 \mathrm{wk} \\
\mathrm{NR}\end{array}$ & $\begin{array}{c}3 \text { sets of } 8-12 \\
\text { repetitions at } 35-70 \% \\
\text { of MVC } \\
\text { NR }\end{array}$ & $\begin{array}{l}\mathrm{NR} \\
\mathrm{NR}\end{array}$ & $\begin{array}{l}95 \\
\text { NR }\end{array}$ & $\begin{array}{l}95 \\
N R\end{array}$ \\
\hline $\begin{array}{l}\text { Medina- } \\
\text { Perez, } 2016^{53}\end{array}$ & $\begin{array}{c}\text { Passive } \\
\text { control } \\
\text { (not defined } \\
\text { usual } \\
\text { activity) }\end{array}$ & $\begin{array}{l}\text { Int: } 38 \\
\text { Con: } 39\end{array}$ & $\begin{array}{l}20 \\
20\end{array}$ & $\begin{array}{l}50 \\
50\end{array}$ & $\begin{array}{l}45.6 \\
41.3\end{array}$ & $\begin{array}{l}100 \\
100\end{array}$ & $\begin{array}{l}3.9 \\
4.2\end{array}$ & $\begin{array}{l}10.9 \\
10.4\end{array}$ & $\begin{array}{l}\text { NR } \\
\text { NR }\end{array}$ & $\begin{array}{c}\text { Supervised } \\
\text { NR }\end{array}$ & $\begin{array}{c}2 d / w k * 12 w k \\
N R\end{array}$ & $\begin{array}{c}3 \text { sets of } 4-10 \\
\text { repetitions at } 40-70 \% \\
\text { of MVC } \\
\text { NR }\end{array}$ & $\begin{array}{l}\mathrm{NR} \\
\mathrm{NR}\end{array}$ & $\begin{array}{l}95 \\
\text { NR }\end{array}$ & $\begin{array}{l}50 \\
N R\end{array}$ \\
\hline $\begin{array}{l}\text { Moradi, } \\
2015^{52}\end{array}$ & $\begin{array}{c}\text { Passive } \\
\text { control } \\
\text { (not defined } \\
\text { usual } \\
\text { activity) }\end{array}$ & $\begin{array}{l}\text { Int: } 10 \\
\text { Con: } 10\end{array}$ & $\begin{array}{c}8 \\
10\end{array}$ & $\begin{array}{l}0 \\
0\end{array}$ & $\begin{array}{l}34.4 \\
33.1\end{array}$ & $\begin{array}{l}62 \\
60\end{array}$ & $\begin{array}{l}3.0 \\
3.0\end{array}$ & $\begin{array}{l}8.1 \\
6.5\end{array}$ & $\begin{array}{l}N R \\
N R\end{array}$ & $\begin{array}{c}\text { Supervised } \\
\text { NR }\end{array}$ & $\begin{array}{c}2 d / w k * 8 w k \\
N R\end{array}$ & $\begin{array}{c}1 \text { set of } 6-15 \text { repetitions } \\
\text { at } 50-80 \% \text { of } 1 \text { RM } \\
\text { NR }\end{array}$ & $\begin{array}{l}30 \\
N R\end{array}$ & $\begin{array}{l}100 \\
N R\end{array}$ & $\begin{array}{l}80 \\
N R\end{array}$ \\
\hline $\begin{array}{l}\text { Inspiratory } \\
\text { muscle } \\
\text { training }\end{array}$ & & & & & & & & & & & & & & & \\
\hline $\begin{array}{l}\text { Mutluay, } \\
2007^{43}\end{array}$ & $\begin{array}{c}\text { Passive } \\
\text { control } \\
\text { (not defined }\end{array}$ & $\begin{array}{l}\text { Int: } 20 \\
\text { Con: } 20\end{array}$ & $\begin{array}{l}20 \\
20\end{array}$ & $\begin{array}{l}60 \\
60\end{array}$ & $\begin{array}{l}40.3 \\
38.1\end{array}$ & $\begin{array}{l}20 \\
40\end{array}$ & $\begin{array}{l}4.8 \\
4.2\end{array}$ & $\begin{array}{l}9.8 \\
9.0\end{array}$ & $\begin{array}{l}\text { Indiv } \\
\text { NR }\end{array}$ & $\begin{array}{c}\text { Unsupervised } \\
\text { NR }\end{array}$ & $\begin{array}{c}7 d / w w^{*} 6 w k \\
N R\end{array}$ & $\begin{array}{l}N R \\
N R\end{array}$ & $\begin{array}{l}\mathrm{NR} \\
\mathrm{NR}\end{array}$ & $\begin{array}{l}94 \\
\text { NR }\end{array}$ & $\begin{array}{l}94 \\
\text { NR }\end{array}$ \\
\hline
\end{tabular}




\begin{tabular}{|c|c|c|c|c|c|c|c|c|c|c|c|c|c|c|c|}
\hline \multirow[b]{2}{*}{ Fry, $2007^{42 *}$} & $\begin{array}{c}\text { usual } \\
\text { activity) }\end{array}$ & & & & & & & & & & & & & & \\
\hline & $\begin{array}{l}\text { Passive } \\
\text { control } \\
\text { (not defined } \\
\text { usual } \\
\text { activity) }\end{array}$ & $\begin{array}{l}\text { Int: } 23 \\
\text { Con: } 23\end{array}$ & $\begin{array}{l}20 \\
21\end{array}$ & $\begin{array}{l}91 \\
74\end{array}$ & $\begin{array}{c}50 \\
46.2\end{array}$ & $\begin{array}{l}50 \\
75\end{array}$ & $\begin{array}{l}3.96 \\
3.36\end{array}$ & $\begin{array}{l}\text { NR } \\
\text { NR }\end{array}$ & $\begin{array}{l}\text { Indiv } \\
\text { NR }\end{array}$ & $\begin{array}{c}\text { Unsupervised } \\
\text { NR }\end{array}$ & $\begin{array}{c}7 d / w k^{*} 10 w k \\
N R\end{array}$ & $\begin{array}{c}3 \text { sets of } 15 \text { repetitions } \\
\text { at 30\% of pretest MIP } \\
\text { NR }\end{array}$ & $\begin{array}{l}\text { NR } \\
\text { NR }\end{array}$ & $\begin{array}{l}81 \\
N R\end{array}$ & $\begin{array}{l}70 \\
\text { NR }\end{array}$ \\
\hline Combined & & & & & & & & & & & & & & & \\
\hline $\begin{array}{l}\text { Aerobic and } \\
\text { resistance }\end{array}$ & & & & & & & & & & & & & & & \\
\hline $\begin{array}{l}\text { Bjarnottir, } \\
2007^{58}\end{array}$ & $\begin{array}{c}\text { Passive } \\
\text { control } \\
\text { (not defined } \\
\text { usual } \\
\text { activity) }\end{array}$ & $\begin{array}{l}\text { Int : } 11 \\
\text { Con: } 12\end{array}$ & $\begin{array}{c}6 \\
10\end{array}$ & $\begin{array}{l}50 \\
80\end{array}$ & $\begin{array}{l}38.7 \\
36.1\end{array}$ & 100 & $\begin{array}{l}2.1 \\
1.8\end{array}$ & $\begin{array}{l}8.7 \\
8.3\end{array}$ & $\begin{array}{l}\text { NR } \\
\text { NR }\end{array}$ & $\begin{array}{c}\text { Supervised } \\
\text { NR }\end{array}$ & $\begin{array}{c}3 d / w k * 5 w k \\
N R\end{array}$ & $\begin{array}{c}55 \% \text { of } \mathrm{VO}_{2 \text { peak }} \\
\mathrm{NR}\end{array}$ & $\begin{array}{l}60 \\
\text { NR }\end{array}$ & $\begin{array}{l}80 \\
N R\end{array}$ & $\begin{array}{l}44 \\
N R\end{array}$ \\
\hline Cakit, $2010^{54}$ & $\begin{array}{l}\text { Comparator } \\
\text { (strength } \\
\text { and balance } \\
\text { exs) } \\
\text { Passive } \\
\text { control } \\
\text { (not defined } \\
\text { usual } \\
\text { activity) }\end{array}$ & $\begin{array}{l}\text { Int 1: } 15 \\
\text { Int 2: } 15 \\
\text { Con: } 15\end{array}$ & $\begin{array}{c}14 \\
10 \\
9\end{array}$ & $\begin{array}{l}60 \\
80 \\
66\end{array}$ & $\begin{array}{l}36.4 \\
43.0 \\
35.5\end{array}$ & $N R$ & $N R$ & $\begin{array}{l}9.2 \\
6.2 \\
6.6\end{array}$ & $\begin{array}{l}\text { Group } \\
\text { Indiv } \\
\text { NR }\end{array}$ & $\begin{array}{c}\text { Supervised } \\
\text { Unsupervised } \\
\text { NR }\end{array}$ & $\begin{array}{c}2 d / w k * 8 w k \\
2 d / w k * 8 w k \\
N R\end{array}$ & $\begin{array}{c}15 \text { sets of } 2 \text { min at } 40 \% \\
\text { of TMW }+2 \text { min at } 30- \\
40 \mathrm{~W} \\
\text { NR } \\
\text { NR }\end{array}$ & $\begin{array}{l}90 \\
30 \\
\text { NR }\end{array}$ & $\begin{array}{l}93 \\
60 \\
\mathrm{NR}\end{array}$ & $\begin{array}{l}87 \\
40 \\
N R\end{array}$ \\
\hline Carter, $2014^{55}$ & $\begin{array}{l}\text { Passive } \\
\text { control } \\
\text { (not defined } \\
\text { usual } \\
\text { activity) }\end{array}$ & $\begin{array}{l}\text { Int: } 60 \\
\text { Con: } 60\end{array}$ & $\begin{array}{l}50 \\
49\end{array}$ & $\begin{array}{l}72 \\
72\end{array}$ & $\begin{array}{c}45.7 \\
46\end{array}$ & $\begin{array}{l}78 \\
85\end{array}$ & $\begin{array}{l}3.8 \\
3.8\end{array}$ & $\begin{array}{l}8.4 \\
9.2\end{array}$ & $\begin{array}{c}\text { Group } \\
\text { NR }\end{array}$ & $\begin{array}{l}\text { Supervised } \\
\text { Unsupervised }\end{array}$ & $\begin{array}{c}2 \mathrm{~d} / \mathrm{wk} * 12 \mathrm{wk} \\
\text { NR }\end{array}$ & $\begin{array}{c}5 \text { sets of } 3-4 \mathrm{~min} \\
\text { intervals at } 50-69 \% \text { of } \\
\mathrm{HR}_{\text {peak }} \text { interspersed with } \\
2 \text { min rest } \\
\mathrm{NR}\end{array}$ & $\begin{array}{l}60 \\
N R\end{array}$ & $\begin{array}{l}90 \\
\mathrm{NR}\end{array}$ & $\begin{array}{l}75 \\
\text { NR }\end{array}$ \\
\hline $\begin{array}{l}\text { Hansen, } \\
2015^{87 *}\end{array}$ & $\begin{array}{c}\text { Passive } \\
\text { control } \\
\text { (not defined } \\
\text { usual } \\
\text { activity) }\end{array}$ & $\begin{array}{l}\text { Int: } 23 \\
\text { Con: } 13\end{array}$ & $\begin{array}{l}16 \\
11\end{array}$ & $\begin{array}{l}63 \\
55\end{array}$ & $\begin{array}{l}46.0 \\
48.0\end{array}$ & $\begin{array}{l}75 \\
55\end{array}$ & $\begin{array}{l}3.0 \\
3.0\end{array}$ & $\mathrm{NR}$ & $\begin{array}{l}N R \\
N R\end{array}$ & $\begin{array}{c}\text { Supervised } \\
\text { NR }\end{array}$ & $\begin{array}{c}5 d / 2 w k * 24 w k \\
N R\end{array}$ & $\begin{array}{l}\text { NR } \\
\text { NR }\end{array}$ & $\begin{array}{c}1 \times 6-3 \times 10 \\
N R\end{array}$ & $\begin{array}{l}90 \\
\mathrm{NR}\end{array}$ & $\begin{array}{l}63 \\
N R\end{array}$ \\
\hline $\begin{array}{l}\text { Hojjatollah, } \\
2012^{49}\end{array}$ & $\begin{array}{l}\text { Passive } \\
\text { control } \\
\text { (not defined } \\
\text { usual } \\
\text { activity) }\end{array}$ & $\begin{array}{l}\text { Int: } 10 \\
\text { Con: } 10\end{array}$ & $\begin{array}{l}10 \\
10\end{array}$ & $\begin{array}{l}0 \\
0\end{array}$ & $\begin{array}{l}\text { NR } \\
\text { NR }\end{array}$ & NR & NR & NR & $\begin{array}{l}\text { NR } \\
\text { NR }\end{array}$ & $\begin{array}{c}\text { Supervised } \\
\text { NR }\end{array}$ & $\begin{array}{c}3 \mathrm{~d} / \mathrm{wk} * 8 \mathrm{wk} \\
\text { NR }\end{array}$ & $\begin{array}{c}3 \text { sets of } 10 \text { repetitions } \\
\text { at } 40-50 \% \text { of } 1 \mathrm{RM} \\
\text { NR }\end{array}$ & $\begin{array}{c}60-90 \\
\text { NR }\end{array}$ & $\begin{array}{l}100 \\
N R\end{array}$ & $\begin{array}{l}100 \\
N R\end{array}$ \\
\hline
\end{tabular}




\begin{tabular}{|c|c|c|c|c|c|c|c|c|c|c|c|c|c|c|c|}
\hline $\begin{array}{l}\text { Kerling, } \\
2015^{88}\end{array}$ & $\begin{array}{l}\text { Active } \\
\text { control } \\
\text { (cycle } \\
\text { endurance) }\end{array}$ & $\begin{array}{l}\text { Int: } 38 \\
\text { Con: } 39\end{array}$ & $\begin{array}{l}19 \\
18\end{array}$ & $\begin{array}{l}80 \\
66\end{array}$ & $\begin{array}{l}42.3 \\
45.6\end{array}$ & NR & $\begin{array}{l}2.6 \\
3.1\end{array}$ & NR & $N R$ & $\begin{array}{l}\text { Supervised } \\
\text { Supervised }\end{array}$ & $\begin{array}{l}2 d / w k^{*} 12 w k \\
2 d / w k^{*} 12 w k\end{array}$ & $\begin{array}{c}50 \% \text { of } \mathrm{PPO} \text { and } 2 \text { sets } \\
\text { of } 10-15 \text { repetitions at } \\
10-15 \mathrm{RM} \\
50 \% \text { of PPO }\end{array}$ & $\begin{array}{l}40 \\
40\end{array}$ & $\begin{array}{l}92 \\
92\end{array}$ & $\begin{array}{l}58 \\
55\end{array}$ \\
\hline $\begin{array}{l}\text { Tallner, } \\
2016^{40}\end{array}$ & $\begin{array}{c}\text { Passive } \\
\text { control } \\
\text { (not defined } \\
\text { waitlist usual } \\
\text { activity) }\end{array}$ & $\begin{array}{l}\text { Int: } 59 \\
\text { Con: } 67\end{array}$ & $\begin{array}{l}49 \\
59\end{array}$ & $\begin{array}{l}75 \\
75\end{array}$ & $\begin{array}{l}40.9 \\
40.7\end{array}$ & $\begin{array}{l}88 \\
85\end{array}$ & $\begin{array}{l}2.7 \\
2.7\end{array}$ & $\begin{array}{l}9.8 \\
9.2\end{array}$ & $\begin{array}{l}\text { Indiv } \\
\text { NR }\end{array}$ & $\begin{array}{c}\text { Unsupervised } \\
\text { NR }\end{array}$ & $\begin{array}{c}3 d / w k^{*} 12 w k \\
N R\end{array}$ & $\begin{array}{l}\text { NR } \\
\text { NR }\end{array}$ & $\begin{array}{l}\text { NR } \\
\text { NR }\end{array}$ & $\begin{array}{l}73 \\
N R\end{array}$ & $\begin{array}{l}61 \\
\text { NR }\end{array}$ \\
\hline $\begin{array}{l}\text { Wens, } 2015^{89} \\
\text { (HIIT) }\end{array}$ & $\begin{array}{l}\text { Comparator } \\
\text { (continuous } \\
\text { training) } \\
\text { Passive } \\
\text { control } \\
\text { (not defined } \\
\text { "sedentary" } \\
\text { control) }\end{array}$ & $\begin{array}{l}\text { Int } 1: 12 \\
\text { Int } 2: 11 \\
\text { Con : } 11\end{array}$ & $\begin{array}{l}12 \\
11 \\
11\end{array}$ & $\begin{array}{l}59 \\
55 \\
82\end{array}$ & $\begin{array}{l}43.0 \\
47.0 \\
47.0\end{array}$ & $\begin{array}{l}73 \\
73 \\
83\end{array}$ & $\begin{array}{l}2.3 \\
2.7 \\
2.5\end{array}$ & NR & NR & $\begin{array}{l}\text { Supervised } \\
\text { Supervised } \\
\text { NR }\end{array}$ & $\begin{array}{c}5 d / 2 w k * 12 w k \\
5 d / 2 w k * 12 w k \\
N R\end{array}$ & $\begin{array}{c}80-100 \% \text { of } H R_{\max } \text { and } \\
1-2 \text { sets of } 10-20 \\
\text { repetitions at } 10-20 \mathrm{RM} \\
80-90 \% \text { of } H R_{\max } \text { and } 1- \\
2 \text { sets of } 10-20 \\
\text { repetitions at } 10-20 \mathrm{RM} \\
\mathrm{NR}\end{array}$ & $\begin{array}{c}\text { Aerobic: } 10- \\
15 \\
\text { Aerobic: 6- } \\
20 \\
\text { NR }\end{array}$ & $\begin{array}{l}90 \\
90 \\
\text { NR }\end{array}$ & $\begin{array}{l}90 \\
90 \\
\text { NR }\end{array}$ \\
\hline $\begin{array}{l}\text { Wens, } \\
2015^{46 *}\end{array}$ & $\begin{array}{c}\text { Passive } \\
\text { control } \\
\text { (not defined } \\
\text { usual } \\
\text { activity) }\end{array}$ & $\begin{array}{l}\text { Int: } 30 \\
\text { Con: } 15\end{array}$ & $\begin{array}{l}29 \\
15\end{array}$ & $\begin{array}{l}59 \\
53\end{array}$ & $\begin{array}{l}48.0 \\
49.0\end{array}$ & $\begin{array}{l}59 \\
73\end{array}$ & $\begin{array}{l}3.25 \\
3.36\end{array}$ & NR & NR & $\begin{array}{c}\text { Supervised } \\
\text { NR }\end{array}$ & $\begin{array}{c}5 d / 2 w k * 24 w k \\
N R\end{array}$ & $\begin{array}{l}\text { NR } \\
\text { NR }\end{array}$ & $\begin{array}{c}10-36 \\
N R\end{array}$ & $\begin{array}{l}90 \\
\text { NR }\end{array}$ & $\begin{array}{l}87 \\
\text { NR }\end{array}$ \\
\hline $\begin{array}{l}\text { Treadmill } \\
\text { walking / } \\
\text { cycle } \\
\text { ergometer+ } \\
\text { Pilates }\end{array}$ & & & & & & & & & & & & & & & \\
\hline Ozkul, $2018^{90}$ & $\begin{array}{l}\text { Passive } \\
\text { control } \\
\text { (relaxation } \\
\text { exs) }\end{array}$ & $\begin{array}{l}\text { Int: } 21 \\
\text { Con: } 20\end{array}$ & $\begin{array}{l}18 \\
18\end{array}$ & $\begin{array}{l}78 \\
78\end{array}$ & $\begin{array}{l}34.5 \\
34.0\end{array}$ & $\begin{array}{l}100 \\
100\end{array}$ & $\begin{array}{l}1.0 \\
1.0\end{array}$ & $\begin{array}{l}4.0 \\
4.0\end{array}$ & $\begin{array}{l}N R \\
N R\end{array}$ & $\begin{array}{c}\text { Supervised } \\
\text { NR }\end{array}$ & $\begin{array}{c}3 x / w k * 8 w k s \\
N R\end{array}$ & $\begin{array}{c}60-80 \% \text { of } \mathrm{HR}_{\max } \text { and } \\
10-20 \text { repetitions in } \\
\text { Pilates } \\
\text { NR }\end{array}$ & $\begin{array}{c}\text { Aerobic: } 30 \\
\text { and Pilates: } \\
60 \\
\text { NR }\end{array}$ & $\begin{array}{l}85 \\
\text { NR }\end{array}$ & $\begin{array}{l}73 \\
N R\end{array}$ \\
\hline $\begin{array}{l}\text { circuit } \\
\text { resistance } \\
\text { training and } \\
\text { aquatic } \\
\text { aerobic } \\
\text { training for } 3 \\
\text { weeks then } \\
23 \text { weeks } \\
\text { home }\end{array}$ & & & & & & & & & & & & & & & \\
\hline
\end{tabular}


Exercise adherence and drop-out in MS

\begin{tabular}{|c|c|c|c|c|c|c|c|c|c|c|c|c|c|c|c|}
\hline $\begin{array}{l}\text { resistance } \\
\text { and aerobic } \\
\text { of choice }\end{array}$ & & & & & & & & & & & & & & & \\
\hline $\begin{array}{l}\text { Romberg, } \\
2004^{45 *}\end{array}$ & $\begin{array}{c}\text { Passive } \\
\text { control } \\
\text { (not defined } \\
\text { usual } \\
\text { activity) }\end{array}$ & $\begin{array}{l}\text { Int: } 49 \\
\text { Con: } 50\end{array}$ & $\begin{array}{l}45 \\
46\end{array}$ & $\begin{array}{l}64 \\
64\end{array}$ & $\begin{array}{l}43.8 \\
43.9\end{array}$ & NR & $\begin{array}{l}2.0 \\
2.5\end{array}$ & $\begin{array}{l}6.0 \\
5.5\end{array}$ & $\begin{array}{l}\text { Indiv } \\
\text { NR }\end{array}$ & $\begin{array}{l}\text { Supervised } \\
3 w k s ; \\
\text { unsupervised } \\
23 \text { wks } \\
\text { NR }\end{array}$ & $\begin{array}{c}50 \mathrm{~min} \\
4-5 d / w k * 26 w k \\
N R\end{array}$ & $\begin{array}{l}\text { NR } \\
\text { NR }\end{array}$ & $\begin{array}{l}\text { NR } \\
\text { NR }\end{array}$ & $\begin{array}{r}93 \\
N R\end{array}$ & $\begin{array}{l}85 \\
\text { NR }\end{array}$ \\
\hline \multicolumn{16}{|l|}{$\begin{array}{l}\text { Self-selected } \\
\text { leg cycle } \\
\text { ergometry, } \\
\text { treadmill } \\
\text { walking, and } \\
\text { recumbent } \\
\text { stepping } \\
\text { exercise+ LL } \\
\text { resistance } \\
\text { training and } \\
\text { balance } \\
\text { exercises }\end{array}$} \\
\hline $\begin{array}{l}\text { Sandroff, } \\
2017^{91}\end{array}$ & $\begin{array}{l}\text { Active } \\
\text { control } \\
\text { (stretching } \\
\text { and toning } \\
\text { exs) }\end{array}$ & $\begin{array}{l}\text { Int: } 43 \\
\text { Con: } 40\end{array}$ & $\begin{array}{l}32 \\
30\end{array}$ & $\begin{array}{l}84 \\
87\end{array}$ & $\begin{array}{l}49.8 \\
51.2\end{array}$ & NR & $\begin{array}{l}\text { PDDS } \\
4.0 \\
3.0\end{array}$ & $N R$ & $\begin{array}{l}\text { Indiv } \\
\text { NR }\end{array}$ & $\begin{array}{c}\text { Supervised } \\
\text { NR }\end{array}$ & $\begin{array}{c}30-60 \min \\
3 d / w k^{*} 24 w k \\
N R\end{array}$ & $\begin{array}{c}40--60 \% \text { of } \mathrm{VO}_{2 \text { peak }} \text { and } \\
40-70 \% \text { of } 1 \mathrm{RM} \\
\mathrm{NR}\end{array}$ & $\begin{array}{c}30-60 \\
\text { NR }\end{array}$ & $\begin{array}{l}81 \\
84\end{array}$ & $\begin{array}{l}60 \\
63\end{array}$ \\
\hline \multicolumn{16}{|l|}{ Other } \\
\hline \multicolumn{16}{|l|}{ Pilates } \\
\hline Duff, $2018^{92}$ & $\begin{array}{c}\text { Passive } \\
\text { control } \\
\text { (massage) }\end{array}$ & $\begin{array}{l}\text { Int: } 15 \\
\text { Con: } 15\end{array}$ & $\begin{array}{l}15 \\
15\end{array}$ & $\begin{array}{l}80 \\
73\end{array}$ & $\begin{array}{l}45.7 \\
45.1\end{array}$ & $\begin{array}{l}93 \\
73\end{array}$ & $\begin{array}{c}\text { PDDS } \\
2.1 \\
2.3\end{array}$ & NR & $\begin{array}{c}\text { Group } \\
\text { Indivi } \\
\text { dual }\end{array}$ & $\begin{array}{l}\text { Supervised } \\
\text { Supervised }\end{array}$ & $\begin{array}{l}3 d / w k^{*} 12 w k \\
1 d / w k^{*} 12 w k\end{array}$ & $\begin{array}{l}\text { NR } \\
\text { NR }\end{array}$ & $\begin{array}{c}50-60 \mathrm{~min} \\
60 \mathrm{~min}\end{array}$ & $\begin{array}{l}85 \\
N R\end{array}$ & $\begin{array}{l}85 \\
N R\end{array}$ \\
\hline
\end{tabular}


Exercise adherence and drop-out in MS

\begin{tabular}{|c|c|c|c|c|c|c|c|c|c|c|c|c|c|c|c|}
\hline \multirow{3}{*}{ Fox, $2016^{41}$} & $\begin{array}{l}\text { Comparator } \\
\text { and passive }\end{array}$ & Int 1: 33 & 33 & 85 & 53.9 & 39 & \multirow{3}{*}{$N R$} & 13.2 & Indiv & Supervised & $1 d / w^{*} 12 w k$ & \multirow{3}{*}{ NR } & 30 & 66 & 56 \\
\hline & control & Int 2: 35 & 32 & 71 & 54.6 & 37 & & 13.9 & Indiv & Supervised & 1d/wk*12wk & & 30 & 84 & 72 \\
\hline & $\begin{array}{l}\text { (relaxation } \\
\text { exs) }\end{array}$ & Con: 32 & 29 & 66 & 53.8 & 38 & & 12.4 & NR & NR & NR & & NR & 92 & 72 \\
\hline $\begin{array}{l}\text { Kalron, } \\
2017^{57}\end{array}$ & $\begin{array}{c}\text { Active } \\
\text { control } \\
\text { (standard } \\
\text { physiotherap } \\
\text { y) }\end{array}$ & $\begin{array}{l}\text { Int: } 25 \\
\text { Con: } 25\end{array}$ & $\begin{array}{l}22 \\
23\end{array}$ & $\begin{array}{l}61 \\
65\end{array}$ & $\begin{array}{l}44.3 \\
42.9\end{array}$ & 100 & $\begin{array}{l}4.3 \\
4.1\end{array}$ & $\begin{array}{l}12.4 \\
11.3\end{array}$ & $\begin{array}{l}\text { Indiv } \\
\text { Indiv }\end{array}$ & $\begin{array}{l}\text { Supervised } \\
\text { Supervised }\end{array}$ & $\begin{array}{l}1 d / w k^{*} 12 w k \\
1 d / w k^{*} 12 w k\end{array}$ & NR & $\begin{array}{l}30 \\
30\end{array}$ & $\begin{array}{l}83 \\
83\end{array}$ & $\begin{array}{l}73 \\
76\end{array}$ \\
\hline \multicolumn{16}{|l|}{ Physiotherapy } \\
\hline $\begin{array}{l}\text { Garrett, } \\
2012^{9}\end{array}$ & $\begin{array}{c}\text { Comparators } \\
\text { (yoga)(fitnes } \\
\text { s instructor) } \\
\text { Passive } \\
\text { control } \\
\text { (not defined } \\
\text { usual } \\
\text { activity) }\end{array}$ & $\begin{array}{l}\text { Int1 :80 } \\
\text { Int2: } 77 \\
\text { Int 3: } 86 \\
\text { Con: } 71\end{array}$ & $\begin{array}{l}63 \\
63 \\
67 \\
49\end{array}$ & $\begin{array}{l}79 \\
70 \\
67 \\
88\end{array}$ & $\begin{array}{l}51.7 \\
49.6 \\
50.3 \\
48.8\end{array}$ & 58 & NR & $\begin{array}{c}9.8 \\
11.6 \\
10.5 \\
10.6\end{array}$ & $\begin{array}{l}\text { Group } \\
\text { Group } \\
\text { Group } \\
\text { NR }\end{array}$ & $\begin{array}{c}\text { Supervised } \\
\text { Supervised } \\
\text { Supervised } \\
\text { NR }\end{array}$ & $\begin{array}{c}1-2 \mathrm{~d} / \mathrm{wk}^{*} 10 \mathrm{wk} \\
\text { Resistance } 2-3 / \mathrm{wk} \\
\text { Aerobic } \\
1 \mathrm{~d} / \mathrm{wk}^{*} 10 \mathrm{wk} \\
1 \mathrm{~d} / \mathrm{wk}^{*} 10 \mathrm{wk} \\
\text { NR }\end{array}$ & $\begin{array}{c}\text { Resistance: } 3 \text { sets of } 12 \\
\text { repetitions at } 12 \mathrm{RM}+ \\
\text { Aerobic: at } 65 \% \text { of } \\
H R_{\max } \\
\mathrm{NR} \\
\mathrm{NR} \\
\mathrm{NR}\end{array}$ & $\begin{array}{c}\text { Resistance: } \\
\text { NR, Aerobic } \\
30 \\
\text { NR } \\
\text { NR } \\
\text { NR }\end{array}$ & $\begin{array}{l}81 \\
73 \\
78 \\
\text { NR }\end{array}$ & $\begin{array}{l}64 \\
60 \\
61 \\
\text { NR }\end{array}$ \\
\hline $\begin{array}{l}\text { Hogan, } \\
2014^{93}\end{array}$ & $\begin{array}{l}\text { Comparators } \\
\text { (1:1 physio) } \\
\text { (yoga) } \\
\text { Passive } \\
\text { control } \\
\text { (usual } \\
\text { activity) }\end{array}$ & $\begin{array}{l}\text { Int 1: } 66 \\
\text { Int 2: } 45 \\
\text { Int 3: } 16 \\
\text { Con: } 19\end{array}$ & $\begin{array}{l}48 \\
35 \\
13 \\
15\end{array}$ & $\begin{array}{l}63 \\
57 \\
62 \\
87\end{array}$ & $\begin{array}{l}57.0 \\
52.0 \\
58.0 \\
49.0\end{array}$ & $\begin{array}{l}27 \\
20 \\
31 \\
33\end{array}$ & NR & $\begin{array}{l}18 \\
13 \\
15 \\
10\end{array}$ & $\begin{array}{l}\text { Group } \\
\text { Indiv } \\
\text { Group } \\
\text { NR }\end{array}$ & $\begin{array}{c}\text { Supervised } \\
\text { Supervised } \\
\text { Supervised } \\
\text { NR }\end{array}$ & $\begin{array}{c}1 \mathrm{~d} / \mathrm{wk}^{*} 10 w k \\
1 \mathrm{~d} / \mathrm{wk}^{*} 10 w k \\
1 \mathrm{~d} / \mathrm{wk}^{*} 10 w k \\
\text { NR }\end{array}$ & NR & $\begin{array}{l}60 \mathrm{~min} \\
60 \mathrm{~min} \\
60 \mathrm{~min} \\
60 \mathrm{~min}\end{array}$ & $\begin{array}{l}80 \\
90 \\
80 \\
\text { NR }\end{array}$ & $\begin{array}{l}58 \\
70 \\
65 \\
\text { NR }\end{array}$ \\
\hline $\begin{array}{l}\text { Conroy, } \\
2018^{38} \\
\text { (with internet } \\
\text { programme) }\end{array}$ & $\begin{array}{c}\text { Active } \\
\text { control } \\
\text { (paper home } \\
\text { exercise } \\
\text { programme) }\end{array}$ & $\begin{array}{l}\text { Int: } 26 \\
\text { Con: } 25\end{array}$ & $\begin{array}{c}16 \\
8\end{array}$ & $\begin{array}{l}44 \\
63\end{array}$ & $\begin{array}{l}50.4 \\
54.3\end{array}$ & $\begin{array}{l}25 \\
50\end{array}$ & $\begin{array}{c}\text { PDDS } \\
4.4 \\
3.3\end{array}$ & $\begin{array}{l}14.2 \\
14.7\end{array}$ & $\begin{array}{l}\text { Indiv } \\
\text { Indiv }\end{array}$ & $\begin{array}{l}\text { Unsupervised } \\
\text { Unsupervised }\end{array}$ & $\begin{array}{c}7 d / w k^{*} 26 w k \\
N R\end{array}$ & NR & NR & $\begin{array}{l}50 \\
N R\end{array}$ & $\begin{array}{l}31 \\
N R\end{array}$ \\
\hline Aquatherapy & & & & & & & & & & & & & & & \\
\hline
\end{tabular}




\begin{tabular}{|c|c|c|c|c|c|c|c|c|c|c|c|c|c|c|c|}
\hline $\begin{array}{l}\text { Sutherland, } \\
2001^{94}\end{array}$ & $\begin{array}{c}\text { Passive } \\
\text { control } \\
\text { (not defined } \\
\text { usual } \\
\text { activity) }\end{array}$ & $\begin{array}{l}\text { Int: } 11 \\
\text { Con: } 11\end{array}$ & $\begin{array}{l}11 \\
11\end{array}$ & $\begin{array}{l}54 \\
54\end{array}$ & $\begin{array}{l}47.2 \\
45.4\end{array}$ & NR & NR & $\begin{array}{l}7.0 \\
7.0\end{array}$ & NR & $\begin{array}{c}\text { Supervised } \\
\text { NR }\end{array}$ & $\begin{array}{c}3 x / w k^{*} 10 w k \\
N R\end{array}$ & NR & $\begin{array}{l}45 \\
\text { NR }\end{array}$ & $\begin{array}{l}90 \\
\text { NR }\end{array}$ & $\begin{array}{l}90 \\
N R\end{array}$ \\
\hline
\end{tabular}

Legend: *main study reported, also reported in following articles: 2009* Dalgas 2010, Dalgas 2010a, Dalgas 2013; Collett 2010* also in Feltham 2013;

Hansen 2015* also in Hansen 2015a, Fry 20017* also in Pfalzer 2011; Kjolhede 2015* also in Kjolhede 2017; Romberg 2004* also in Romberg 2005, Surakka 2004; Wens 2015* also in Wens 2016.

${ }^{+}$Adherence calculated to include drop-outs (drop-outs assigned $0 \%$ adherence)

Abbreviations: PPO: Peak power output achieved during incremental exercise test to exhaustion, $\mathrm{VO}_{2 \text { max }}$ maximal oxygen consumption, $\mathrm{VO}_{2 \text { peak: }}$ peak oxygen consumption, RM: repetition maximum, BW: body weight, MVC: Maximal voluntary contraction, MIP: Maximal inspiratory pressure, TMW:

Tolerated maximum workload, W: Watts; PDDS: Patient determined disease steps; Indiv: Individual; NR: not reported. 


\subsection{Reporting of adherence and drop-out}

Authors reported adherence in a variety of ways including: the number or proportion of participants attending a particular number of sessions $(n=7)$; the number or proportion of participants attending all sessions $(n=6)$; the total number of prescribed exercise sessions attended $(n=2)$; mean number of sessions attended by participants (either as a single average $(n=7)$ or as an average accompanied by a measure of variation $(n=19))$.

With respect to adherence to the exercise protocol (content adherence), thirtyfive of the forty-one studies did not provide any detail to confirm completion of the exercise program as prescribed. A further five studies reported only very briefly on intervention completion, by statements such as: "the intervention was completed as prescribed" 91, 93; participants "completed all the scheduled training sessions" 86; "affirmed full compliance with the programme" ${ }^{43}$ or that "the intervention schedule was completed". ${ }^{48}$ Only one study gave additional, although limited details, by reporting that adherence to the program was successful, with $95 \%$ of exercise sessions completed and participants in the exercise group successfully increasing the weight in their vests by $0.5 \%$ to $1.0 \%$ of body weight during the 8 -week intervention. ${ }^{11}$

Reported adherence to an exercise intervention (exercise comparator or active control) ranged from $50 \%{ }^{38,48}-100 \% 47,49$ during the intervention period and $20 \%^{50}-88 \%{ }^{51}$ in the three studies reporting this during follow up. Seventy eight percent of included interventions reported adherence of $80 \%$ or more. Eighty two percent of these were supervised interventions. In two studies, flexibility regarding time frame for completing the intervention was allowed within the protocol in order to 
attain $80 \%$ adherence. ${ }^{12,52}$ Methods used to measure adherence were stated in eleven studies, some of which included more than one method, and included session attendance monitoring $(n=5)$, self-report diary or logbook (paper; $n=9$; electronic diary $n=1)$ or activity tracker $(n=1)$.

The pooled estimate of adherence was 0.87 (95\% Cl 0.83 to 0.90$)$, as illustrated in Figure 2. This estimate represents the proportion of participants reported as adherent to the intervention when adherence data were combined in a meta-analysis. The I-squared statistic was 57\% (95\% Cl $46.4 \%$ to $76.2 \%)$ indicating a moderate-to-high degree of heterogeneity. 
Figure 2. Pooled adherence (excluding drop-outs) according to intervention type

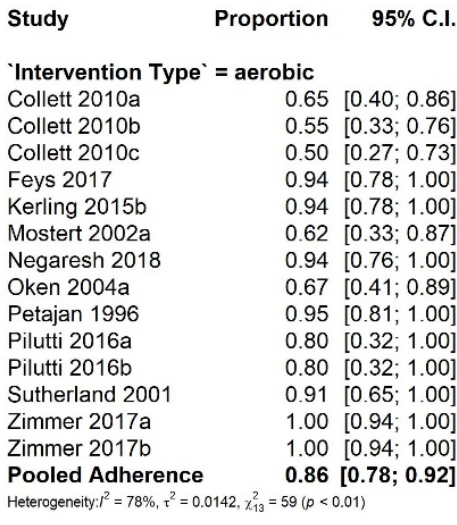

$\begin{array}{lr}\text { 'Intervention Type }=\text { resistance } \\ \text { Cakit 2010b } & 0.60[0.28 ; 0.89] \\ \text { Conroy 2018a } & 0.50[0.25 ; 0.75] \\ \text { Dalgas 2009 } & 1.00[0.89 ; 1.00] \\ \text { DeBolt 2004 } & 0.95[0.79 ; 1.00] \\ \text { Dodd 2011 } & 0.92[0.80 ; 0.99] \\ \text { Fimland 2010a } & 1.00[0.77 ; 1.00] \\ \text { Hosseini 2018a } & 1.00[0.80 ; 1.00] \\ \text { Hogan 2014a } & 0.79[0.66 ; 0.90] \\ \text { Harvey 1999b } & 0.67[0.24 ; 0.99] \\ \text { Kjolhede 2015 } & 0.94[0.76 ; 1.00] \\ \text { Manca 2017a } & 0.93[0.74 ; 1.00] \\ \text { Manca 2017b } & 0.93[0.74 ; 1.00] \\ \text { Medina-Perez 2014 } & 0.97[0.86 ; 1.00] \\ \text { Medina-Perez 2016 } & 0.95[0.80 ; 1.00] \\ \text { Moradi 2015 } & 1.00[0.80 ; 1.00] \\ \text { Pooled Adherence } & \mathbf{0 . 9 1}[0.85 ; 0.96] \\ \text { Heterogeneity }: I^{2}=58 \%, \tau^{2}=0.0142, \chi_{14}^{2}=33(p<0.01)\end{array}$

'Intervention Type = combined

$\begin{array}{lc}\text { 'Intervention Type }=\text { combined } \\ \text { Bjarnottir 2007 } & 0.83[0.41 ; 1.00] \\ \text { Cakit 2010a } & 0.93[0.72 ; 1.00] \\ \text { Carter 2014 } & 0.90[0.80 ; 0.97] \\ \text { Hojjatollah 2012 } & 1.00[0.83 ; 1.00] \\ \text { Harvey 1999a } & 0.67[0.24 ; 0.99] \\ \text { Hansen 2015 } & 0.88[0.66 ; 1.00] \\ \text { Garrett 2012a } & 0.81[0.70 ; 0.90] \\ \text { Kalron 2017b } & 0.83[0.64 ; 0.96] \\ \text { Kerling 2015a } & 0.89[0.71 ; 1.00] \\ \text { Ozkul 2018 } & 0.83[0.62 ; 0.98] \\ \text { Romberg 2004 } & 0.93[0.84 ; 0.99] \\ \text { Sandroff 2017a } & 0.81[0.66 ; 0.93] \\ \text { Tallner 2016 } & 0.72[0.56 ; 0.86] \\ \text { Wens 2015a } & 0.92[0.68 ; 1.00] \\ \text { Wens 2015b } & 0.91[0.65 ; 1.00] \\ \text { Wens 2015 } & 0.90[0.75 ; 0.99] \\ \text { Garrett 2012b } & 0.78[0.67 ; 0.87] \\ \text { Hogan 2014b } & 0.91[0.79 ; 0.99] \\ \text { Pooled Adherence } & \mathbf{0 . 8 7}[0.81 ; 0.92] \\ \text { Heterogeneity: } l^{2}=5 \%, \tau^{2}=0.0142, \chi_{17}^{2} & 18(p=0.40)\end{array}$

Heterogeneity $I^{2}=5 \%, \tau^{2}=0.0142, \chi_{17}^{2}=18(\rho=0$
Intervention Type' $=$ other

$\begin{array}{lr}\text { Duff 2018 } & 0.87[0.64 ; 1.00] \\ \text { Forsberg 2016 } & 0.86[0.72 ; 0.96] \\ \text { Fox 2016a } & 0.67[0.50 ; 0.82] \\ \text { Hosseini 2018b } & 1.00[0.80 ; 1.00] \\ \text { Fry 2007 } & 0.80[0.59 ; 0.95] \\ \text { Kalron 2017a } & 0.82[0.63 ; 0.96] \\ \text { Mutluay 2007 } & 0.95[0.80 ; 1.00] \\ \text { Oken 2004b } & 0.68[0.47 ; 0.86] \\ \text { Sandroff 2017b } & 0.83[0.68 ; 0.95] \\ \text { Garrett 2012c } & 0.73[0.61 ; 0.83] \\ \text { Hogan 2014c } & 0.77[0.50 ; 0.96] \\ \text { Fox 2016b } & 0.84[0.69 ; 0.95] \\ \text { Pooled Adherence } & \mathbf{0 . 8 2}[\mathbf{0 . 7 4} \mathbf{0 . 8 9}] \\ \text { Heterogeneity }:^{2}=24 \%, \tau^{2}=0.0142, \chi_{11}^{2}=14(p=0.21)\end{array}$

Heterogeneity: $I^{2}=24 \%, \tau^{2}=0.0142, x_{11}^{2}=14(p=0.21)$

Pooled Adherence $\quad 0.87[0.83 ; 0.90]$

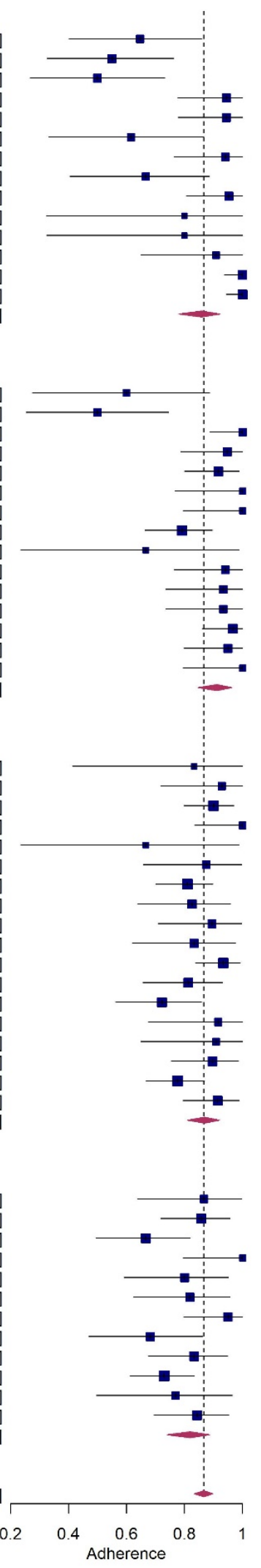


The reported drop-outs from exercise interventions ranged from $0 \%{ }^{47}$ to $47 \% 53$ during the intervention period and $0-2740 \%$ at follow up. There was a lack of consistency in reporting adherence and drop-out numbers and reasons for drop-out. This was the case regardless as to whether or not studies reported adherence at follow up. For instance, some studies excluded people who did not reach a predefined cut-off level of adherence to an intervention, such as $75 \%^{53}$ or $80 \%{ }^{46,50}$ of sessions attended and instead reported them as drop-outs.

When adherence was re-calculated to include drop-outs, the pooled adherence was $0.73(95 \% \mathrm{Cl} 0.68$ to 0.78$)$ and I-squared was $73 \%(95 \% \mathrm{Cl} 66.0 \%$ to $84.7 \%$ ) as illustrated in figure 3 .

Insert figure 3 
Figure 3. Pooled adherence (including drop-outs) according to intervention type

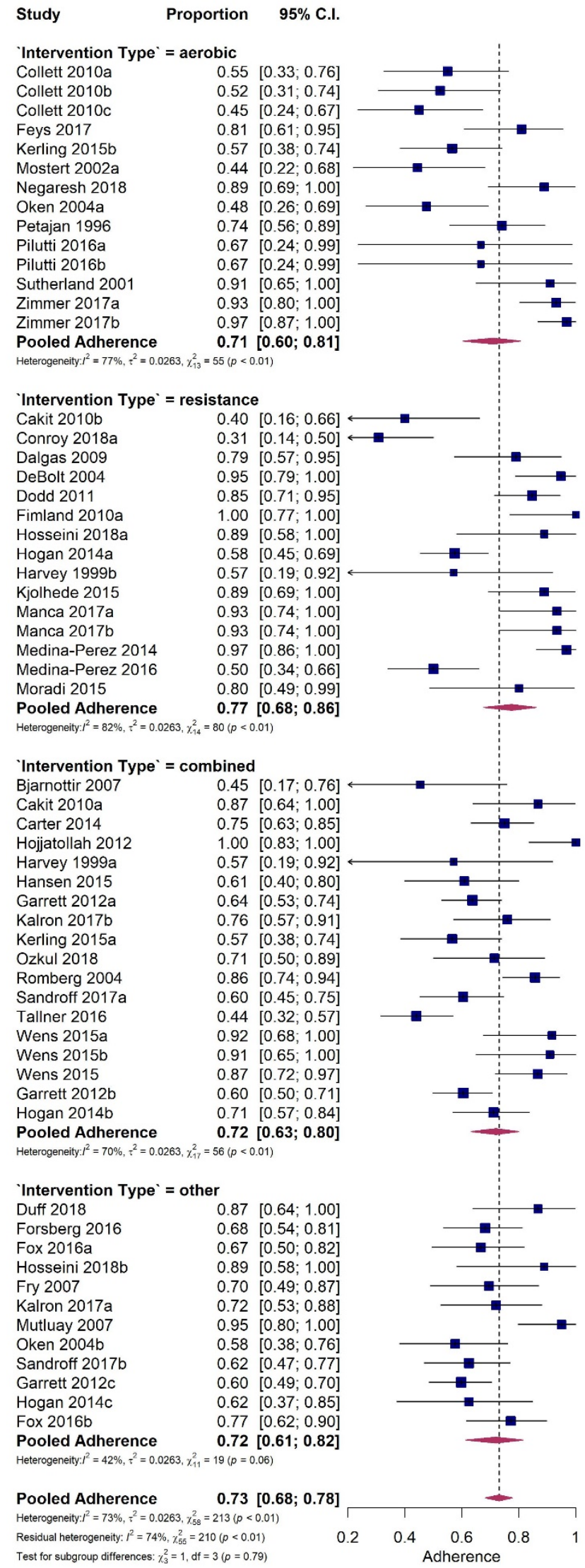




\subsection{Moderators}

Only mean age (estimate $=-0.009$, standard error $=0.003, p=0.013$ ), proportion of female participants (estimate $=-0.003$, standard error $=0.001, p=0.017$ ), and duration of intervention (estimate $=-0.007$, standard error $=0.003, p=0.045$ ) all showed statistically significant inverse associations with adherence and together explained $31 \%$ of the adherence heterogeneity.

A range of potential strategies to enhance adherence have been suggested. ${ }^{17,18}$ Our moderator analysis however suggested that there was not a significant association with adherence in relation to the use of behavioural interventions or a home exercise component. Fifteen studies ${ }^{9,11,12,38-45,54-57}$ incorporated a home exercise element, eight of which were home based interventions. Fifteen studies $9,11,12,38,40-45,54-56,58,59$ included a behavioural intervention component. These were reported as goal setting, ${ }^{12,55}$ use of an activity tracker, ${ }^{12}$ telephone support, ${ }^{11,42,45}$ face to face support, ${ }^{11}$ peer support, ${ }^{12}$ social support, ${ }^{55,59}$ education regarding benefits of exercise $9,42,55,58$ and log or workbooks. ${ }^{41,44}$ Only one ${ }^{55}$ of the studies described the theoretical background of the behavioural component.

\subsection{Quality}

The mean TESTEX score of the included studies reporting adherence was 7.5/15. Details of the individual scores can be found in table 2 . Sixty six $(70 \%)$ studies scored below 10 points, which although not designed to be a cutoff point, is 
suggested by the scale's authors as indicative of poor study design and/ or reporting (personal communication). TESTEX score was not found to be a moderator of adherence.

Insert table 2 here 
Table 2: TESTEX scores of all included studies

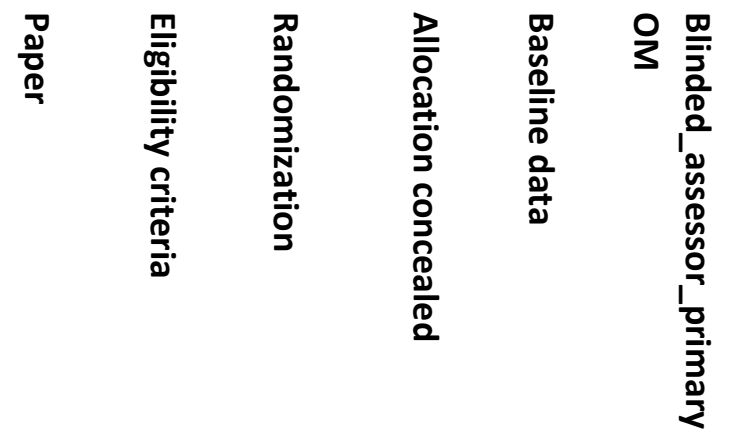

$\begin{array}{llllll}\text { Ahmadi 2010 } & 0 & 0 & 1 & 1 & 0 \\ \text { Ahmadi 2010 } & 1 & 0 & 1 & 1 & 0 \\ \text { Ahmadi 2013 } & 1 & 0 & 1 & 1 & 0 \\ \text { Ahmadi 2013 } & 1 & 0 & 1 & 1 & 0 \\ \text { Aidar 2018 } & 0 & 1 & 0 & 0 & 0 \\ \text { Amiri 2018 } & 0 & 0 & 0 & 0 & 0 \\ \text { Aydin 2014 } & 0 & 1 & 0 & 1 & 0 \\ \text { Bansi 2012 } & 1 & 1 & 0 & 1 & 1 \\ \text { Bansi 2013 } & 1 & 1 & 0 & 1 & 0 \\ \text { Barrett 2009 } & 1 & 1 & 0 & 1 & 0 \\ * \text { Bjarnadottir } & 1 & 0 & 1 & 1 & 1 \\ \text { 2007 } & & & & \end{array}$
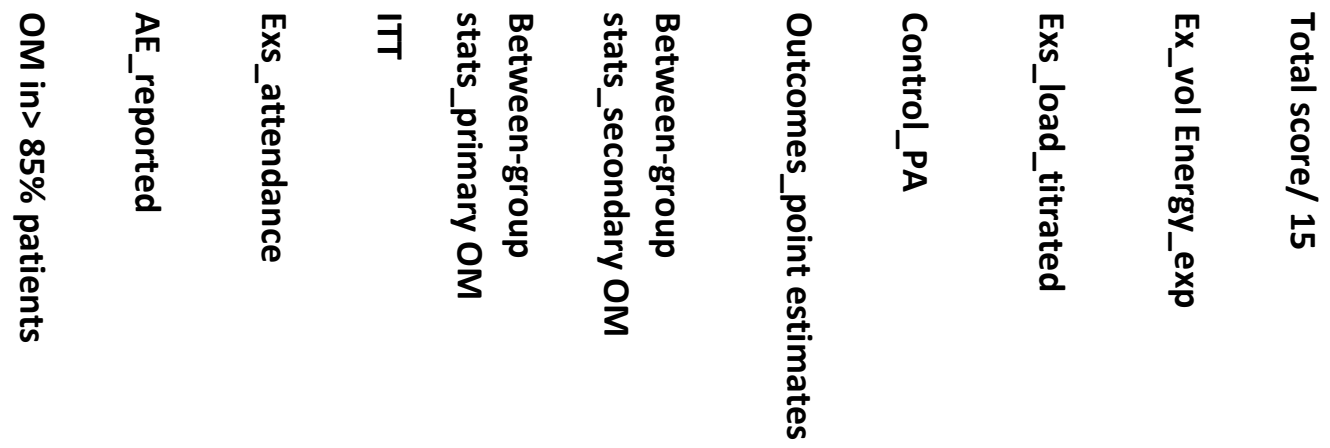

$\begin{array}{llllllllll}0 & 0 & 0 & 0 & 0 & 1 & 0 & 0 & 0 & 4 \\ 0 & 0 & 0 & 0 & 0 & 1 & 0 & 0 & 0 & 5 \\ 0 & 0 & 0 & 0 & 0 & 1 & 0 & 0 & 1 & 6 \\ 0 & 0 & 0 & 0 & 0 & 1 & 0 & 0 & 1 & 6 \\ 0 & 0 & 0 & 0 & 0 & 1 & 0 & 0 & 0 & 3 \\ 0 & 0 & 0 & 0 & 0 & 1 & 0 & 0 & 0 & 2 \\ 0 & 0 & 0 & 0 & 0 & 1 & 0 & 0 & 0 & 4 \\ 0 & 0 & 0 & 0 & 0 & 1 & 0 & 0 & 1 & 7 \\ 0 & 0 & 0 & 1 & 1 & 1 & 0 & 0 & 1 & 8 \\ 1 & 0 & 0 & 1 & 1 & 1 & 0 & 0 & 0 & 7 \\ 1 & 0 & 0 & 0 & 0 & 1 & 0 & 1 & 1 & 8\end{array}$




\begin{tabular}{|c|c|c|c|c|c|c|c|c|c|c|c|c|c|c|c|}
\hline Bulguroglu 2017 & 1 & 0 & 0 & 1 & $1^{* *}$ & 1 & 1 & 0 & 0 & 0 & 0 & 1 & 0 & 1 & 0 \\
\hline *Cakit 2010 & 1 & 1 & 1 & 1 & 1 & 0 & 0 & 1 & 0 & 1 & 1 & 1 & 0 & 1 & 1 \\
\hline *Carter 2014 & 1 & 0 & 1 & 1 & 0 & 1 & 1 & 1 & 0 & 1 & 1 & 1 & 0 & 1 & 0 \\
\hline $\begin{array}{l}\text { Castro-Sánchez } \\
2011\end{array}$ & 1 & 0 & 1 & 1 & 1 & 1 & 1 & 0 & 0 & 1 & 1 & 1 & 0 & 0 & 0 \\
\hline *Collett 2010 & 1 & 1 & 1 & 1 & 1 & 0 & 1 & 1 & 1 & 1 & 1 & 1 & 1 & 1 & 1 \\
\hline *Conroy 2018 & 1 & 0 & 1 & 1 & 1 & 0 & 0 & 0 & 0 & 1 & 1 & 1 & 0 & 0 & 0 \\
\hline *Dalgas 2009 & 1 & 1 & 1 & 1 & 0 & 0 & 0 & 1 & 0 & 1 & 1 & 1 & 0 & 1 & 1 \\
\hline Dalgas 2010 & 1 & 0 & 1 & 1 & 0 & 0 & 0 & 1 & 0 & 0 & 0 & 1 & 0 & 1 & 1 \\
\hline Dalgas 2010 & 1 & 1 & 1 & 1 & 0 & 1 & 0 & 1 & 0 & 0 & 1 & 1 & 0 & 1 & 1 \\
\hline Dalgas 2013 & 1 & 0 & 1 & 1 & 0 & 0 & 0 & 0 & 0 & 0 & 1 & 1 & 0 & 1 & 1 \\
\hline *DeBolt 2004 & 1 & 0 & 1 & 0 & 0 & 1 & 0 & 1 & 0 & 1 & 1 & 1 & 0 & 0 & 0 \\
\hline Dettmers 2009 & 1 & 0 & 0 & 1 & 0 & 1 & 0 & 0 & 0 & 1 & 0 & 1 & 0 & 0 & 0 \\
\hline *Dodd 2011 & 1 & 1 & 1 & 1 & 1 & 1 & 1 & 1 & 1 & 1 & 1 & 1 & 1 & 1 & 0 \\
\hline *Duff 2018 & 1 & 1 & 1 & 1 & 1 & 1 & 1 & 1 & 1 & 1 & 1 & 1 & 0 & 0 & 0 \\
\hline Ebrahimi 2012 & 1 & 0 & 0 & 1 & $1^{* *}$ & 1 & 0 & 0 & 0 & 0 & 1 & 1 & 0 & 1 & 1 \\
\hline Eftekhari 2012 & 1 & 0 & 1 & 1 & 0 & 1 & 1 & 0 & 0 & 0 & 1 & 0 & 0 & 0 & 0 \\
\hline Eftekhari 2018 & 1 & 0 & 0 & 1 & 0 & 0 & 0 & 0 & 0 & 0 & 1 & 1 & 0 & 0 & 0 \\
\hline $\begin{array}{l}\text { Escudero-Uribe } \\
2017\end{array}$ & 1 & 1 & 1 & 1 & $1^{* *}$ & 1 & 1 & 0 & 0 & 0 & 1 & 1 & 0 & 0 & 0 \\
\hline
\end{tabular}




\begin{tabular}{|c|c|c|c|c|c|c|c|c|c|c|c|c|c|c|c|}
\hline Feltham 2013 & 1 & 1 & 1 & 1 & 1 & 0 & 0 & 1 & 0 & 0 & 0 & 0 & 0 & 1 & 1 \\
\hline *Feys 2017 & 1 & 0 & 0 & 1 & 0 & 0 & 1 & 1 & 1 & 1 & 1 & 1 & 0 & 0 & 1 \\
\hline *Fimland 2010 & 1 & 0 & 1 & 1 & 0 & 1 & 1 & 1 & 0 & 0 & 1 & 0 & 0 & 1 & 1 \\
\hline *Forsberg 2016 & 1 & 1 & 1 & 1 & 1 & 0 & 1 & 1 & 0 & 1 & 1 & 1 & 0 & 0 & 0 \\
\hline *Fox 2016 & 1 & 1 & 1 & 1 & 1 & 0 & 1 & 1 & 1 & 1 & 1 & 1 & 0 & 0 & 0 \\
\hline Frevel 2015 & 1 & 1 & 1 & 1 & 0 & 1 & 0 & 0 & 0 & 1 & 1 & 1 & 0 & 0 & 0 \\
\hline *Fry 2007 & 1 & 0 & 1 & 1 & $1 * *$ & 1 & 0 & 1 & 0 & 0 & 1 & 1 & 0 & 0 & 1 \\
\hline Gandolfi, 2014 & 1 & 1 & 1 & 1 & 1 & 1 & 1 & 0 & 1 & 1 & 1 & 1 & 0 & 0 & 0 \\
\hline *Garrett, 2012 & 0 & 0 & 0 & 0 & 1 & 0 & 0 & 0 & 0 & 1 & 1 & 1 & 0 & 1 & 1 \\
\hline Golzari, 2010 & 1 & 0 & 0 & 1 & 0 & 0 & 0 & 0 & 0 & 0 & 0 & 1 & 0 & 1 & 0 \\
\hline Hansen, 2015i & 0 & 1 & 1 & 1 & 1 & 0 & 0 & 1 & 0 & 1 & 1 & 1 & 0 & 1 & 0 \\
\hline *Hansen, 2015ii & 0 & 1 & 1 & 1 & 1 & 0 & 1 & 1 & 0 & 1 & 1 & 1 & 0 & 1 & 0 \\
\hline *Harvey, 1999 & 1 & 1 & 0 & 1 & 0 & 1 & 0 & 1 & 0 & 0 & 0 & 0 & 0 & 0 & 0 \\
\hline $\begin{array}{l}\text { Hassanpour- } \\
\text { Dekho, } 2016\end{array}$ & 1 & 1 & 0 & 0 & $1^{* *}$ & 1 & 0 & 0 & 0 & 0 & 0 & 1 & 0 & 0 & 0 \\
\hline Hayes, 2011 & 1 & 0 & 1 & 1 & 0 & 1 & 1 & 1 & 0 & 0 & 0 & 1 & 1 & 1 & 0 \\
\hline Hebert, 2011 & 1 & 1 & 1 & 1 & 1 & 1 & 1 & 1 & 1 & 1 & 1 & 1 & 0 & 0 & 1 \\
\hline Heine, 2017 & 1 & 1 & 0 & 0 & 1 & 0 & 0 & 1 & 1 & 1 & 1 & 1 & 1 & 1 & 1 \\
\hline *Hogan, 2014 & 1 & 1 & 1 & 0 & $1^{* *}$ & 0 & 0 & 1 & 0 & 0 & 0 & 1 & 0 & 1 & 0 \\
\hline
\end{tabular}


*Hojjatollah

2012

\begin{tabular}{|c|c|c|c|c|c|c|c|c|c|c|c|c|c|c|c|}
\hline *Hosseini, 2018 & 1 & 1 & 0 & 1 & 0 & 1 & 0 & 0 & 0 & 0 & 0 & 1 & 0 & 0 & 0 \\
\hline *Kalron, 2017 & 1 & 1 & 0 & 1 & $1 * *$ & 1 & 1 & 1 & 0 & 0 & 0 & 1 & 0 & 1 & 0 \\
\hline Kargarfard, 2012 & 1 & 1 & 1 & 1 & $1 * *$ & 0 & 1 & 0 & 1 & 0 & 0 & 1 & 0 & 0 & 0 \\
\hline *Kerling, 2015 & 1 & 0 & 1 & 1 & 1 & 0 & 0 & 1 & 1 & 1 & 1 & 1 & 0 & 1 & 1 \\
\hline *Kjolhede, 2015 & 1 & 1 & 1 & 0 & 0 & 1 & 0 & 1 & 1 & 0 & 0 & 1 & 0 & 1 & 1 \\
\hline Kjolhede, 2017 & 0 & 1 & 1 & 0 & $1 * *$ & 0 & 0 & 0 & 0 & 0 & 0 & 1 & 0 & 0 & 0 \\
\hline Klefbeck, 2003 & 1 & 0 & 0 & 1 & 0 & 1 & 0 & 0 & 0 & 0 & 0 & 0 & 0 & 1 & 1 \\
\hline Kooshiar, 2014 & 1 & 1 & 1 & 0 & 0 & 1 & 0 & 0 & 0 & 0 & 0 & 1 & 0 & 0 & 0 \\
\hline Kucuk, 2016 & 1 & 1 & 0 & 1 & 0 & 0 & 0 & 0 & 0 & 0 & 0 & 1 & 0 & 0 & 0 \\
\hline Magnani, 2016 & 1 & 1 & 0 & 1 & 0 & 0 & 0 & 0 & 0 & 1 & 1 & 0 & 0 & 1 & 1 \\
\hline *Manca, 2017 & 1 & 1 & 1 & 1 & $1 * *$ & 0 & 1 & 1 & 0 & 0 & 0 & 1 & 0 & 0 & 1 \\
\hline $\begin{array}{l}\text { *Medina-Perez, } \\
2014\end{array}$ & 1 & 0 & 1 & 1 & 0 & 1 & 1 & 1 & 0 & 1 & 1 & 1 & 0 & 1 & 1 \\
\hline $\begin{array}{l}\text { *Medina-Perez, } \\
2016\end{array}$ & 1 & 0 & 1 & 1 & $1 * *$ & 0 & 1 & 1 & 0 & 0 & 1 & 1 & 0 & 1 & 1 \\
\hline $\begin{array}{l}\text { Mokhtarzade, } \\
2017\end{array}$ & 1 & 0 & 1 & 1 & 0 & 1 & 0 & 0 & 0 & 0 & 0 & 1 & 0 & 1 & 1 \\
\hline *Moradi, 2015 & 1 & 1 & 0 & 1 & $1 * *$ & 0 & 1 & 0 & 0 & 0 & 0 & 1 & 0 & 1 & 1 \\
\hline Mori, 2011 & 1 & 0 & 1 & 1 & 1 & 0 & 0 & 0 & 0 & 0 & 0 & 1 & 0 & 0 & 0 \\
\hline
\end{tabular}




\begin{tabular}{|c|c|c|c|c|c|c|c|c|c|c|c|c|c|c|c|}
\hline *Mostert, 2002 & 1 & 0 & 1 & 0 & 0 & 0 & 0 & 1 & 0 & 0 & 0 & 0 & 1 & 0 & 0 \\
\hline *Mutluay, 2007 & 1 & 1 & 0 & 1 & $1 * *$ & 1 & 0 & 1 & 0 & 0 & 1 & 1 & 0 & 0 & 0 \\
\hline $\begin{array}{l}\text { Najafidoulatabad } \\
\text {, } 2014\end{array}$ & 1 & 0 & 0 & 0 & 0 & 0 & 0 & 0 & 0 & 0 & 0 & 1 & 0 & 0 & 0 \\
\hline *Negaresh, 2018 & 1 & 1 & 0 & 0 & 1 & 1 & 1 & 1 & 0 & 1 & 1 & 1 & 0 & 1 & 1 \\
\hline *Oken, 2004 & 1 & 0 & 1 & 1 & 1 & 0 & 1 & 1 & 0 & 1 & 0 & 1 & 0 & 0 & 0 \\
\hline *Ozkul, 2018 & 1 & 1 & 1 & 1 & 1 & 1 & 0 & 1 & 0 & 1 & 0 & 1 & 0 & 1 & 1 \\
\hline Pazokian, 2013 & 1 & 0 & 0 & 0 & 0 & 0 & 0 & 0 & 0 & 0 & 0 & 1 & 0 & 0 & 0 \\
\hline *Petajan, 1996 & 1 & 0 & 0 & 1 & $1 * *$ & 1 & 0 & 1 & 0 & 0 & 0 & 1 & 0 & 1 & 1 \\
\hline Pfalzer, 2011 & 1 & 0 & 0 & 1 & $1 * *$ & 0 & 0 & 1 & 0 & 0 & 0 & 1 & 0 & 0 & 0 \\
\hline *Pilutti, 2016 & 1 & 1 & 1 & 0 & 0 & 0 & 1 & 1 & 0 & 1 & 1 & 1 & 1 & 1 & 0 \\
\hline Razazian, 2016 & 1 & 1 & 0 & 1 & $1 * *$ & 1 & 0 & 0 & 0 & 0 & 0 & 1 & 0 & 0 & 0 \\
\hline Riksfjord, 2017 & 1 & 0 & 1 & 0 & 0 & 1 & 1 & 0 & 0 & 1 & 1 & 1 & 0 & 1 & 1 \\
\hline *Romberg, 2004 & 1 & 0 & 0 & 0 & 0 & 0 & 1 & 1 & 1 & 1 & 1 & 1 & 0 & 1 & 0 \\
\hline Romberg, 2005 & 1 & 0 & 0 & 1 & 0 & 1 & 0 & 0 & 1 & 0 & 0 & 1 & 0 & 0 & 0 \\
\hline Samaei, 2016 & 1 & 1 & 1 & 1 & 1 & 1 & 0 & 0 & 1 & 1 & 1 & 1 & 0 & 1 & 1 \\
\hline *Sandroff, 2017 & 1 & 1 & 1 & 1 & 0 & 0 & 0 & 1 & 1 & 1 & 1 & 1 & 1 & 1 & 1 \\
\hline Sangelaji, 2016 & 1 & 0 & 0 & 1 & $1^{* *}$ & 1 & 0 & 0 & 1 & 0 & 0 & 1 & 0 & 1 & 1 \\
\hline Schulz, 2004 & 1 & 0 & 1 & 1 & 0 & 0 & 0 & 0 & 0 & 0 & 0 & 1 & 0 & 0 & 1 \\
\hline Smeltzer, 1996 & 0 & 0 & 1 & 1 & 0 & 0 & 0 & 0 & 0 & 0 & 0 & 0 & 0 & 1 & 0 \\
\hline
\end{tabular}




\begin{tabular}{|c|c|c|c|c|c|c|c|c|c|c|c|c|c|c|c|}
\hline Surakka, 2004 & 1 & 1 & 0 & 1 & 0 & 1 & 0 & 1 & 0 & 0 & 0 & 1 & 0 & 1 & 0 \\
\hline $\begin{array}{l}\text { *Sutherland, } \\
2001\end{array}$ & 1 & 0 & 0 & 1 & 0 & 1 & 0 & 1 & 0 & 0 & 0 & 1 & 0 & 0 & 0 \\
\hline *Tallner, 2016 & 1 & 1 & 1 & 1 & 1 & 0 & 0 & 1 & 0 & 1 & 1 & 1 & 1 & 0 & 0 \\
\hline Tarakci, 2013 & 1 & 0 & 1 & 1 & 1 & 1 & 1 & 0 & 1 & 1 & 1 & 1 & 0 & 0 & 0 \\
\hline Velikonja, 2010 & 0 & 0 & 0 & 0 & $1^{* *}$ & 0 & 0 & 0 & 0 & 0 & 0 & 1 & 0 & 0 & 0 \\
\hline *Wens, 2015i & 1 & 1 & 1 & 1 & 0 & 1 & 1 & 1 & 0 & 1 & 1 & 1 & 0 & 1 & 0 \\
\hline *Wens, 2015ii & 1 & 1 & 1 & 1 & 0 & 1 & 1 & 1 & 1 & 1 & 1 & 1 & 0 & 1 & 1 \\
\hline Wens, 2016 & 1 & 1 & 1 & 1 & 0 & 1 & 1 & 1 & 0 & 1 & 1 & 1 & 0 & 1 & 0 \\
\hline $\begin{array}{l}\text { Westerdahl, } \\
2015\end{array}$ & 1 & 1 & 1 & 1 & 1 & 1 & 1 & 0 & 0 & 1 & 1 & 1 & 0 & 0 & 0 \\
\hline *Zimmer, 2017 & 1 & 0 & 1 & 1 & 1 & 1 & 1 & 1 & 1 & 1 & 1 & 1 & 1 & 0 & 1 \\
\hline
\end{tabular}

Legend: **no primary outcome but assessor blinded. *Study included in meta-analysis.

i/ii Two studies by the same author in the same year in referenced order where included in meta-analysis. 


\section{Discussion}

The results of this systematic review provide a novel synthesis of the measurement and reporting of adherence and drop-out from exercise interventions (both exercise comparators and active controls) in exercise studies in MS. Overall measurement and reporting is poor, with only half $(41 / 81)$ of the identified RCT studies reporting adherence. The lack of data is particularly striking in the follow up period where only three of 81 studies reported whether participants continued to engage in exercise. Hence we cannot ascertain from the existing evidence whether or not pwMS continue to exercise in the medium or longer term following the initiation of an exercise programme. This potentially limits the translation of results to clinical practice, since clinicians cannot make evidence based decisions regarding which exercise approach is most effective in sustaining long-term engagement in exercise; this being known to be required to retain any immediate benefits gained. In addition, only a few weak adherence moderators were identified. It would have also been interesting to determine levels of adherence to the control group intervention, however this data was rarely recorded or reported.

On a more positive note, it is encouraging that adherence to the exercise interventions (based on attendance to supervised sessions and percentage completion of prescribed home programme exercises) was high in the 41 studies which reported adherence during the intervention period, with the majority $(78 \%)$ reporting adherence in excess of $80 \%$. It is noteworthy, that more than $80 \%$ of this data related to attendance at supervised sessions. It is possible however, that this level of adherence is an overestimation given half of the exercise studies did not report adherence which potentially may have been due to poor levels of adherence rather than lack of measurement. During follow up, when supervision had ceased, 
the three studies reporting adherence scored $20,{ }^{50} 36^{40}$ and $88 \%{ }^{51}$ although this data should be interpreted with caution given the very limited number of studies upon which this is based.

Drop-out during intervention ranged from $0-47 \%$ and $0-27 \%$ at follow up. Whilst it is possible that participants may have dropped out for reasons related to the exercise intervention, unfortunately, a lack of consistency and detail in reporting means that it is not possible to determine whether or not this was the case. The limited available data provided wide ranging reasons for drop-out, which include personal circumstances (e.g. family illness) which are not modifiable when considering potential changes to either study or intervention design. Of note, Pilutti et al. ${ }^{60}$ has previously reviewed the literature and reported that the number of adverse events in, and drop-outs from MS exercise groups are comparable to those from the control groups. In addition, the exercise groups in general had a risk reduction of $27 \%$ for having a relapse thus, adverse events and an increased relapse rate are likely not major factors affecting adherence in MS exercise studies.

There are several major findings of this review. Firstly, the variety of adherence definitions reported by the identified studies suggests that trials do not consider adherence in consistent ways. Indeed the focus of a laboratory-based proof of concept intervention study will have different issues related to adherence than a pragmatic community-based physiotherapy intervention. It may therefore be that different definitions are appropriate for different trials, however their definition should clarify the specific elements of adherence that are being addressed. ${ }^{61}$ 
The majority of studies in the review reported the proportions of available sessions attended as the measure of adherence. This data is informative for determining feasibility of the programme, particularly from a service delivery perspective. Another aspect of adherence relates to whether an exercise intervention is completed at the prescribed intensity and/or duration of the protocol (content adherence). This provides information as to whether an adequate training stimulus was received, and whether the prescribed training is achievable for all participants. In this review only one study ${ }^{11}$ was identified that provided any detail on this aspect of adherence and none explicitly reported details of how many people deviated from the prescribed training protocol with respect to intensity and/or duration. As exercise protocol deviations are likely to be present in most studies, future trials should optimally provide data on planned versus actual intensity (such as heart rate data confirming aerobic intensity or loading data from resistance training) and duration of exercise. This also highlights the issue that words often used synonymously may indicate different aspects of adherence, a finding also underlined by other reviews of adherence in different populations. ${ }^{62,63}$ In addition, studies reported adherence of completer participants. Reporting adherence including that of those who dropped out of a study for an intervention related reason would add further transparency and accuracy of intervention adherence. Interestingly the Consensus on Exercise Reporting Template (CERT) only recommend that a detailed description is provided on how adherence to exercise is measured and reported, but do not provide any specific recommendation on how to report adherence. ${ }^{64}$

A second finding is that of how adherence is measured. To our knowledge there is no guidance available regarding the optimal method for measuring 
adherence in exercise studies in people with neurological conditions, although attempts have been made to make such recommendations within the musculoskeletal field. ${ }^{65}$ In our review, the majority of studies measured adherence as session attendance. However, some studies measured adherence via self-report electronic or paper logbooks or self-report diaries. The use of different methods makes comparison between studies or inclusion within meta-analysis difficult. Furthermore, interpretation of the self-report data needs to be considered mindful of the widely recognised issues of over-inflation of exercise reporting, memory recall and social desirability. ${ }^{66}$ Thirdly, without a follow up period post intervention it is not possible to know whether people continued to engage in exercise or if any benefits resulting from a given intervention are maintained long-term, which is a key focus of current research. Only eleven of the studies in this review included such a follow up period and of these only three made comment as to whether exercise adherence continued over this time. The necessity of evaluating long-term follow up to determine whether short-term changes persist is emphasised in the Development and Evaluation of Complex Interventions guidance. ${ }^{67}$ Our review demonstrates that this recommendation is not yet being widely followed.

It is noteworthy that in this review, we have highlighted studies that include comprehensive behavioural interventions as well as those incorporating a component in line with Michie et. al. ${ }^{68}$ such as follow up phone calls or completion of activity logs. Although the benefits of including a behavioural intervention are recognised in the literature, only around a third of studies reported inclusion of such and in the majority of cases the extent of this component appeared to be limited. Although not included in this review, it is encouraging that more recent pilot and 
feasibility studies ${ }^{69-71}$ are seeking to further evaluate the addition of such interventions which may provide useful future insights.

An objective of this review was to identify moderators related to adherence and drop-out during the exercise intervention and at follow up. Of the variables assessed, only age, proportion of females and duration of intervention were the significant moderators. This finding was unexpected. On the basis of clinical experience and studies investigating correlates and determinates of physical activity. ${ }^{72}$ it was anticipated that disease duration and level of disability might also have been significant moderators. So too might programme related factors such as the exercise modality, mode of intervention delivery, and whether or not the programme was supervised, as has been the case in studies in other populations ${ }^{29,73,74}$ however this was not the case. In addition, group allocation may have been a moderator but analysis of this was not possible due to the lack of passive control (usual activity) group adherence data.

As has been the finding of previous systematic reviews of rehabilitation interventions, ${ }^{61,75,76}$ reporting of the study methods and results was not consistently of a high standard. In particular this was with respect to the transparency of reporting and incomplete or inaccurate reporting (such as of drop-out data). This makes both interpretation and implementation of the results more difficult for both researchers and clinicians. It underlines the necessity for authors to more closely follow the Consolidated Standards of Reporting Trials (CONSORT), ${ }^{77}$ CERT $^{64}$ and the Template for Intervention Description and Replication (TIDieR) ${ }^{78}$ guidelines in order 
to facilitate the translation of evidence to practice. In addition, future exercise studies should seek to report their work in line with the criteria for the development and evaluation of complex interventions, ${ }^{79}$ where process evaluation is considered important to exploring issues related to the delivery and uptake of an intervention, such as adherence.

\section{Strengths and limitations}

This is a comprehensive review of adherence to exercise interventions over the last 25 years. Conducted in line with PRISMA guidance and utilising an exercise specific methodological appraisal tool, it provides a robust overview of the MS specific exercise literature. The study, however has several limitations, which include possible bias as studies not published in English were not included. The grey literature was not searched in this systematic review which may be a further limitation. Finally, we did not go back to the original authors for raw data, since the focus of our review was on the measurement and reporting of adherence data. Whilst this may have provided additional information, poor response rates are common when attempting to retrieve such data. ${ }^{80}$ The results should therefore be interpreted in light of these.

\section{Conclusions}

Only half of the existing exercise RCT studies in MS report data on both adherence and drop-out during the intervention period, and it was very rare for this data to be gathered at follow up. In addition, only a few weak moderators of exercise adherence were identified. Researchers are urged to consider clear definitions and 
presentation of adherence data within future studies to enable the clinician to make a balanced cost-benefit decision regarding implementation.

\section{Acknowledgements}

The authors would like to thanks Lorna Burns (information specialist at University of Plymouth) for her help with designing the search strategy.

This research did not receive any specific grant from funding agencies in the public, commercial, or not-for-profit sectors.

\section{Conflict of interest}

Conflict of interest: None.

Appendix A: example search strategy

Appendix B: list of all included studies 


\section{References}

1. Edwards T, Pilutti LA. The effect of exercise training in adults with multiple sclerosis with severe mobility disability: A systematic review and future research directions. Mult Scler Relat Disord. 2017;16:31-39.

2. Heine M, van de Port I, Rietberg MB, van Wegen EE, Kwakkel G. Exercise therapy for fatigue in multiple sclerosis. Cochrane Database Syst Rev. 2015(9):CD009956.

3. Jorgensen M, Dalgas U, Wens I, Hvid LG. Muscle strength and power in persons with multiple sclerosis - A systematic review and meta-analysis. $J$ Neurol Sci. 2017;376:225-241.

4. Latimer-Cheung AE, Pilutti LA, Hicks AL, et al. Effects of exercise training on fitness, mobility, fatigue, and health-related quality of life among adults with multiple sclerosis: a systematic review to inform guideline development. Arch Phys Med Rehabil. 2013;94(9):1800-1828 e1803.

5. Kinnett-Hopkins D, Adamson B, Rougeau K, Motl RW. People with MS are less physically active than healthy controls but as active as those with other chronic diseases: An updated meta-analysis. Mult Scler Relat Disord. $2017 ; 13: 38-43$.

6. Motl R, Sandroff B, Kwakkel G, Dalgas U, Feinstein A, Heesen C et al. Exercise in patients with multiple sclerosis. The Lancet Neurology 2017;16(10):848-856.

7. Feinstein A, Freeman J, Lo AC. Treatment of progressive multiple sclerosis: what works, what does not, and what is needed. The Lancet Neurology. 2015;14(2):194-207. 
8. Riemenschneider M, Hvid LG, Stenager E, Dalgas U. Is there an overlooked "window of opportunity" in MS exercise therapy? Perspectives for early MS rehabilitation. Mult Scler. 2018;24(7):886-894.

9. Garrett M, Hogan N, Larkin A, Saunders J, Jakeman P, Coote S. Exercise in the community for people with minimal gait impairment due to MS: an assessor-blind randomized controlled trial. Mult Scler. 2013;19(6):782-789.

10. Freeman J, Hendrie W, Jarrett L, et al. Assessment of a home-based standing frame programme in people with progressive multiple sclerosis (SUMS): a pragmatic, multi-centre, randomised, controlled trial and cost-effectiveness analysis. The Lancet Neurology. 2019;18(8):736-747.

11. DeBolt LS, McCubbin JA. The effects of home-based resistance exercise on balance, power, and mobility in adults with multiple sclerosis. Arch Phys Med Rehabil. 2004;85(2):290-297.

12. Feys $\mathrm{P}$, Moumdjian L, Van Halewyck F, et al. Effects of an individual 12-week community-located "start-to-run" program on physical capacity, walking, fatigue, cognitive function, brain volumes, and structures in persons with multiple sclerosis. Mult Scler. 2019;25(1):92-103.

13. Zimmer P, Bloch W, Schenk A, et al. High-intensity interval exercise improves cognitive performance and reduces matrix metalloproteinases-2 serum levels in persons with multiple sclerosis: A randomized controlled trial. Mult Scler. 2018;24(12):1635-1644.

14. McAuley E, Motl R, Morris K, et al. Enhancing physical activity adherence and well-being in multiple sclerosis: a randomised controlled trial. Mult Scler. 2007;13(5): 652-659. 
15. Dennett R, Gunn H, Freeman J. Effectiveness of and User Experience With Web-Based Interventions in Increasing Physical Activity Levels in People With Multiple Sclerosis: A Systematic Review. Physical Therapy. 2018;98(8):679_ 690.

16. Emmerson KB, Harding KE, Taylor NF. Providing exercise instructions using multimedia may improve adherence but not patient outcomes: a systematic review and meta-analysis. Clin Rehabil. 2019;33(4):607-618.

17. Casey B, Coote S, Hayes S, Gallagher S. Changing Physical Activity Behavior in People With Multiple Sclerosis: A Systematic Review and MetaAnalysis. Arch Phys Med Rehabil. 2018;99(10):2059-2075.

18. Motl RW, Hubbard EA, Bollaert RE, et al. Randomized controlled trial of an elearning designed behavioral intervention for increasing physical activity behavior in multiple sclerosis. Mult Scler J Exp Trans/ Clin. 2017;3(4):2055217317734886.

19. Motl R, Pekmezi D, Wingo BC. Promotion of physical activity and exercise in multiple sclerosis: Importance of behavioral science and theory. Mult Scler J Exp Transl Clin. 2018;4(3):2055217318786745.

20. Twomey L, Taylor J. Old age and physical capacity: use it or lose it. Aust $\mathrm{J}$ of physiother. 1984;30(4):115-120.

21. Organization WH. Adherence To Long-term Therapies: Evidence for action. 2003.

22. Allen NE, Sherrington C, Suriyarachchi GD, Paul SS, Song J, Canning CG. Exercise and motor training in people with Parkinson's disease: a systematic review of participant characteristics, intervention delivery, retention rates, 
adherence, and adverse events in clinical trials. Parkinsons Dis. 2012;2012:854328.

23. Moher D, Liberati A, Tetzlaff J, Altman D. Preferred Reporting Items for Systematic Reviews and Meta-Analyses: The PRISMA Statement. PLoS Med 6(7): e1000097. 2009.

24. Caspersen C, Powell K, Christenson G. Physical Activity, Exercise, and Physical Fitness: Definitions and Distinctions for Health-Related Research. Public Health Reports. 1985;100(2):126-131.

25. E S. Adherence to long term therapies: evidence for action. In: Geneva: World Health Organisation; 2003.

26. Directive 2001/20/EC of the European Parliament and of the Council of 4 April 2001 on the approximation of the laws, regulations and administrative provisions of the member states relating to the implementation of good clinical practice in the conduct of clinical trials on medicinal products for human use. Med Etika Bioet. 2002;9(1):12-19.

27. Hozo SP, Djulbegovic B, Hozo I. Estimating the mean and variance from the median, range, and the size of a sample. BMC Med Res Methodol. 2005;5:13.

28. Smart NA, Waldron $\mathrm{M}$, Ismail $\mathrm{H}$, et al. Validation of a new tool for the assessment of study quality and reporting in exercise training studies: TESTEX. Int J Evid Based Healthc. 2015;13(1):9-18.

29. McPhate L, Simek EM, Haines TP. Program-related factors are associated with adherence to group exercise interventions for the prevention of falls: a systematic review. Journal of Physiotherapy. 2013;59(2):81-92.

30. Viechtbauer W. Conducting meta-analyses in $\mathrm{R}$ with the metafor package. Journal of Statistical Software. 2010;36(3):1-48. 
31. CoreTeam R. A language and environment for statistical computing. $R$ Foundation for Statistical Computing. Vienna, Austria. 2019.

32. Kurtzke J. Rating neurologic impairment in multiple sclerosis: an expanded disability status scale (EDSS). Neurology. 1983;33(11):1444-1452.

33. Learmonth Y, Motl R, Sandroff B, Pula J, Cadavid D. Validation of patient determined disease steps (PDDS) scale scores in persons with multiple sclerosis. BMC Neurology 2013;13(37).

34. Sharrack B, Hughes R. The Guy's Neurological Disability Scale (GNDS): a new disability measure for multiple sclerosis. Mult Scler. 1999;5(4):223-233.

35. Aidar FJ, Gama de Matos D, de Souza RF, et al. Influence of aquatic exercises in physical condition in patients with multiple sclerosis. $J$ Sports Med Phys Fitness. 2018;58(5):684-689.

36. Klefbeck B, Hamrah Nedjad J. Effect of inspiratory muscle training in patients with multiple sclerosis. Arch Phys Med Rehabil. 2003;84(7):994-999.

37. Pilutti LA, Paulseth JE, Dove C, Jiang S, Rathbone MP, Hicks AL. Exercise Training in Progressive Multiple Sclerosis: A Comparison of Recumbent Stepping and Body Weight-Supported Treadmill Training. Int J MS Care. 2016;18(5):221-229.

38. Conroy SS, Zhan M, Culpepper WJ, 2nd, Royal W, 3rd, Wallin MT. Selfdirected exercise in multiple sclerosis: Evaluation of a home automated telemanagement system. J Telemed Telecare. 2018;24(6):410-419.

39. Hosseini SS, Rajabi H, Sahraian MA, Moradi M, Mehri K, Abolhasani M. Effects of 8-Week Home-Based Yoga and Resistance Training on Muscle Strength, Functional Capacity and Balance in Patients with Multiple Sclerosis: A Randomized Controlled Study. Asian Journal of Sports Medicine. 2018;9(3). 
40. Tallner A, Streber R, Hentschke C, et al. Internet-Supported Physical Exercise Training for Persons with Multiple Sclerosis-A Randomised, Controlled Study. Int J Mol Sci 2016;17.

41. Fox E, Hough A, Creanor S, Gear M, Freeman J. Effects of Pilates-Based Core Stability Training in Ambulant People With Multiple Sclerosis: Multicenter, Assessor-Blinded, Randomized Controlled Trial. Physical Therapy 2016;96(8):1170-1178.

42. Fry DK, Pfalzer LA, Chokshi AR, Wagner MT, Jackson ES. Randomized control trial of effects of a 10 -week inspiratory muscle training program on measures of pulmonary function in persons with multiple sclerosis. $J$ Neurol Phys Ther. 2007;31(4):162-172.

43. Mutluay F, Demir R, Ozyilmaz S, Caglar A, Altintas A, Gurses H. Breathingenhanced upper extremity exercises for patients with multiple sclerosis. Clin Rehabil 2007;21: 595-602.

44. Oken B, Kishiyama S, Zajdel D, et al. Randomized controlled trial of yoga and exercise in multiple sclerosis. Neurology. 2004;62:2058-2064.

45. Romberg A, Virtanen A, Ruutiainen J, et al. Effects of a 6-month exercise program on patients with multiple sclerosis A randomized study. Neurology. 2004;63:2034-2038.

46. Wens I, Hansen D, Verboven K, et al. Impact of 24 Weeks of Resistance and Endurance Exercise on Glucose Tolerance in Persons with Multiple Sclerosis. Am J Phys Med Rehabil. 2015;94(10 Suppl 1):838-847.

47. Fimland MS, Helgerud J, Gruber M, Leivseth G, Hoff J. Enhanced neural drive after maximal strength training in multiple sclerosis patients. Eur J Appl Physiol. 2010;110(2):435-443. 
48. Collett J, Dawes H, Meaney A, et al. Exercise for multiple sclerosis: a singleblind randomized trial comparing three exercise intensities. Mult Scler. 2011;17(5):594-603.

49. Hojjatollah N, Khosrow E, Shirazi Reza R, Monireh M. Effects of Selected Combined Training on Muscle Strength in Multiple Sclerosis Patients. HealthMED 2012;6(4):1258-1264.

50. Dalgas U, Stenager E, Jakobsen J, et al. Resistance training improves muscle strength and functional capacity in multiple sclerosis. Neurology. 2009;73:1487-1484.

51. Kjolhede T, Vissing K, de Place L, et al. Neuromuscular adaptations to longterm progressive resistance training translates to improved functional capacity for people with multiple sclerosis and is maintained at follow-up. Mult Scler. 2015;21(5):599-611.

52. Moradi M, Sahraian MA, Aghsaie A, Kordi MR, Meysamie A, Abolhasani M. Effects of Eight-week Resistance Training Program in Men With Multiple Sclerosis. Asian Journal of Sports Medicine. 2015;6(2).

53. Medina-Perez C, de Souza-Teixeira F, Fernandez-Gonzalo R, HernandezMurua JA, Antonio de Paz-Fernandez J. Effects of high-speed power training on muscle strength and power in patients with multiple sclerosis. J Rehabil Res Dev. 2016;53(3):359-368.

54. Cakit BD, Nacir B, Genc $\mathrm{H}$, et al. Cycling progressive resistance training for people with multiple sclerosis: a randomized controlled study. Am J Phys Med Rehabil. 2010;89(6):446-457.

55. Carter A, Daley A, Humphreys L, et al. Pragmatic intervention for increasing self-directed exercise behaviour and improving important health outcomes in 
people with multiple sclerosis: a randomised controlled trial. Mult Scler. 2014;20(8):1112-1122.

56. Harvey L, Smith AD, Jones R. The Effect of Weighted Leg Raises on Quadriceps Strength, EMG Parameters and Functional Activities in People with Multiple Sclerosis. Physiotherapy. 1999;85(3):154-161.

57. Kalron A, Rosenblum U, Frid L, Achiron A. Pilates exercise training vs. physical therapy for improving walking and balance in people with multiple sclerosis: a randomized controlled trial. Clin Rehabil. 2017;31(3):319-328.

58. Bjarnadottir O, Konradsdottir A, K R, E O. Multiple sclerosis and brief moderate exercise. A randomised study. Multiple Sclerosis 2007. 2007;13:776-782.

59. Dodd KJ, Taylor NF, Shields N, Prasad D, McDonald E, Gillon A. Progressive resistance training did not improve walking but can improve muscle performance, quality of life and fatigue in adults with multiple sclerosis: a randomized controlled trial. Mult Scler. 2011;17(11):1362-1374.

60. Pilutti LA, Platta ME, Motl RW, Latimer-Cheung AE. The safety of exercise training in multiple sclerosis: a systematic review. J Neurol Sci. 2014;343(12):3-7.

61. Hawley-Hague H, Horne M, Skelton DA, Todd C. Review of how we should define (and measure) adherence in studies examining older adults' participation in exercise classes. BMJ Open. 2016;6(6):e011560.

62. Ezzat AM, MacPherson K, Leese J, Li LC. The effects of interventions to increase exercise adherence in people with arthritis: a systematic review. Musculoskeletal Care. 2015;13(1):1-18. 
63. Frost R, Levati S, McClurg D, Brady M, Williams B. What Adherence Measures Should Be Used in Trials of Home-Based Rehabilitation Interventions? A Systematic Review of the Validity, Reliability, and Acceptability of Measures. Arch Phys Med Rehabil. 2017;98(6):1241-1256 e1245.

64. Slade S, Dionne C, Underwood M, Buchbinder R. Consensus on Exercise reporting Template (CERT): Explanation and Elaboration Statement. British Journal of Sports Medicine. 2016;50(23):1428-1437.

65. Holden MA, Haywood K, Potia T, Gee M, McLean S. Recommendations for exercise adherence measures in musculoskeletal settings: a systematic review and consensus meeting (protocol). Systematic Reviews 2014;3(10).

66. Jansons PS, Haines TP, O'Brien L. Interventions to achieve ongoing exercise adherence for adults with chronic health conditions who have completed a supervised exercise program: systematic review and meta-analysis. Clin Rehabil. 2017;31(4):465-477.

67. Mohler R. Criteria for Reporting the Development and Evaluation of Complex Interventions in healthcare: revised guideline (CReDECI 2). Trials. $2015 ; 16(204)$.

68. Michie S, Ashford S, Sniehotta FF, Dombrowski SU, Bishop A, French DP. A refined taxonomy of behaviour change techniques to help people change their physical activity and healthy eating behaviours: the CALO-RE taxonomy. Psychol Health. 2011;26(11):1479-1498.

69. Coote S, Uszynski M, Herring MP, et al. Effect of exercising at minimum recommendations of the multiple sclerosis exercise guideline combined with 
structured education or attention control education - secondary results of the step it up randomised controlled trial. BMC Neurol. 2017;17(1):119.

70. Hayes S, Uszynski MK, Motl RW, et al. Randomised controlled pilot trial of an exercise plus behaviour change intervention in people with multiple sclerosis: the Step it Up study. BMJ Open. 2017;7(10):e016336.

71. Keytsman C, Van Noten P, Spaas J, Nieste I, Van Asch P, Eijnde BO. Periodized home-based training: A new strategy to improve high intensity exercise therapy adherence in mildly affected patients with Multiple Sclerosis. Mult Scler Relat Disord. 2019;28:91-97.

72. Streber R, Peters S, Pfeifer K. Systematic Review of Correlates and Determinants of Physical Activity in Persons With Multiple Sclerosis. Arch Phys Med Rehabil. 2016;97(4):633-645 e629.

73. Reljic D, Lampe D, Wolf F, Zopf Y, Herrmann HJ, Fischer J. Prevalence and predictors of dropout from high-intensity interval training in sedentary individuals: A meta-analysis. Scand J Med Sci Sports. 2019.

74. Allen N, Song J, Paul SS, et al. Predictors of Adherence to a Falls Prevention Exercise Program for People with Parkinson's Disease." 2(4): 395-401. Movement Disorders Clinical Practice. 2015;2(4):395-401.

75. McLean S, Holden MA, Potia T, et al. Quality and acceptability of measures of exercise adherence in musculoskeletal settings: a systematic review. Rheumatology (Oxford). 2017;56(3):426-438.

76. Jordan JL, Holden MA, Mason EE, Foster NE. Interventions to improve adherence to exercise for chronic musculoskeletal pain in adults. Cochrane Database Syst Rev. 2010(1):CD005956. 
77. Page P, Hoogenboom B, Voight M. Improving the reporting of therapeutic exercise interventions in rehabilitation research. The International Journal of Sports Physical Therapy 2017;12(2):297-304.

78. Hoffmann T, Glasziou P, Boutron I, Milne R, al e. Better reporting of interventions: template for intervention description and replication (TIDieR) checklist and guide. BMJ. 2014;38:1687.

79. Mohler R, Copke S, Meyer G. Criteria for Reporting the Development and Evaluation of Complex Interventions in healthcare: revised guideline (CReDECI 2). Trials. 2015;16(204).

80. Schroll JB, Bero L, Gotzsche PC. Searching for unpublished data for Cochrane reviews: cross sectional study. BMJ. 2013;346:f2231.

81. Mostert S, Kesselring J. Effects of a short-term exercise training program on aerobic fitness, fatigue, health perception and activity level of subjects with multiple sclerosis. Multiple Sclerosis Journal. 2002;8: 161 -168.

82. Negaresh R, Motl R, Mokhtarzade M, et al. Effect of Short-Term Interval Exercise Training on Fatigue, Depression, and Fitness in Normal Weight vs. Overweight Person With Multiple Sclerosis. Explore (NY). 2018;15(2):134141.

83. Petajan J, Gappmaier E, White A, Spencer M, Mino L, Hicks R. Impact of Aerobic Training on Fitness and Qdty of Life in Multiple Sclerosis. Ann Neurol $1996 ; 33: 432-441$.

84. Forsberg A, von Koch L, Nilsagard Y. Effects on Balance and Walking with the CoDuSe Balance Exercise Program in People with Multiple Sclerosis: A Multicenter Randomized Controlled Trial. Mult Scler Int. 2016;2016:7076265. 
85. Manca A, Cabboi MP, Dragone D, et al. Resistance Training for Muscle Weakness in Multiple Sclerosis: Direct Versus Contralateral Approach in Individuals With Ankle Dorsiflexors' Disparity in Strength. Arch Phys Med Rehabil. 2017;98(7):1348-1356 e1341.

86. Medina-Perez C, de Souza-Teixeira F, Fernandez-Gonzalo R, de PazFernandez JA. Effects of a resistance training program and subsequent detraining on muscle strength and muscle power in multiple sclerosis patients. NeuroRehabilitation. 2014;34(3):523-530.

87. Hansen D, Wens I, Keytsman C, Verboven K, Dendale P, Eijnde B. Ventilatory function during exercise in multiple sclerosis and impact of training intervention: cross-sectional and randomized controlled trial. Eur J Phys Rehabil Med 2015;51:557-568.

88. Kerling A, Keweloh K, Tegtbur U, et al. Effects of a Short Physical Exercise Intervention on Patients with Multiple Sclerosis (MS). Int J Mol Sci. 2015;16(7):15761-15775.

89. Wens I, Dalgas U, Vandenabeele F, et al. High Intensity Exercise in Multiple Sclerosis: Effects on Muscle Contractile Characteristics and Exercise Capacity, a Randomised Controlled Trial. PLoS One. 2015;10(9):e0133697.

90. Ozkul C, Guclu-Gunduz A, Irkec C, et al. Effect of combined exercise training on serum brain-derived neurotrophic factor, suppressors of cytokine signaling 1 and 3 in patients with multiple sclerosis. J Neuroimmunol. 2018;316:121129.

91. Sandroff BM, Bollaert RE, Pilutti LA, et al. Multimodal exercise training in multiple sclerosis: A randomized controlled trial in persons with substantial mobility disability. Contemp Clin Trials. 2017;61:39-47. 
92. Duff WRD, Andrushko JW, Renshaw DW, et al. Impact of Pilates Exercise in Multiple Sclerosis: A Randomized Controlled Trial. Int J MS Care. 2018;20(2):92-100.

93. Hogan N, Kehoe M, Larkin A, Coote S. The Effect of Community Exercise Interventions for People with MS Who Use Bilateral Support for Gait. Mult Scler Int. 2014;2014:109142.

94. Sutherland G, Andersen MB, Stoové MA. Can Aerobic Exercise Training Affect Health-Related Quality of Life for People with Multiple Sclerosis? Journal of Sport and Exercise Psychology. 2001;23(2):122-135. 